\title{
Enhancement of carotenoid production by disrupting the C22-sterol desaturase gene (CYP61) in Xanthophyllomyces dendrorhous
}

Iris Loto, María Soledad Gutiérrez, Salvador Barahona, Dionisia Sepúlveda, Pilar Martínez-Moya, Marcelo Baeza, Víctor Cifuentes and Jennifer Alcaíno*

\begin{abstract}
Background: Xanthophyllomyces dendrorhous is a basidiomycetous yeast that synthesizes astaxanthin, which is a carotenoid with a great biotechnological impact. The ergosterol and carotenoid synthesis pathways are derived from the mevalonate pathway, and in both pathways, cytochrome P450 enzymes are involved.

Results: In this study, we isolated and described the X. dendrorhous CYP61 gene, which encodes a cytochrome P450 involved in ergosterol biosynthesis. This gene is composed of nine exons and encodes a 526 amino acid polypeptide that shares significant percentages of identity and similitude with the C22-sterol desaturase, CYP61, from other fungi. Mutants derived from different parental strains were obtained by disrupting the CYP61 gene with an antibiotic selection marker. These mutants were not able to produce ergosterol and accumulated ergosta-5,8, 22-trien-3-ol and ergosta-5,8-dien-3-ol. Interestingly, all of the mutants had a more intense red color phenotype than their respective parental strains. The carotenoid composition was qualitatively and quantitatively analyzed by RP-HPLC, revealing that the carotenoid content was higher in the mutant strains without major changes in their composition. The expression of the HMGR gene, which encodes an enzyme involved in the mevalonate pathway (3-hydroxy-3-methylglutaryl-CoA reductase), was analyzed by RT-qPCR showing that its transcript levels are higher in the CYP61 mutants.
\end{abstract}

Conclusions: These results suggest that in X. dendrorhous, ergosterol regulates HMGR gene expression by a negative feedback mechanism and in this way; it contributes in the regulation of the carotenoid biosynthesis.

Keywords: Xanthophyllomyces dendrorhous, Astaxanthin, Ergosterol, Sterol C22-sterol desaturase, Cytochrome P450

\section{Background}

Xanthophyllomyces dendrorhous is a basidiomycetous carotenogenic yeast and is one of the few known natural sources of xanthophyll astaxanthin (3,3'-dihydroxy- $\beta$, $\beta$-carotene-4-4'-dione) [1-3]. Carotenogenesis may have evolved as a cellular defense mechanism against oxidative damage from reactive oxygen species (ROS) produced by biochemical and photochemical systems [4-6]. Among carotenoids, astaxanthin stands out for its potent antioxidant properties and other beneficial effects on human health [7]. Moreover, this pigment has been widely used in

\footnotetext{
* Correspondence: jalcainog@u.uchile.cl
Laboratorio de Genética. Departamento de Ciencias Ecológicas y Centro de

* Correspondence: jalcainog@u.uchile.cl
Laboratorio de Genética. Departamento de Ciencias Ecológicas y Centro de Biotecnología, Facultad de Ciencias, Universidad de Chile, Las Palmeras 3425, Santiago Casilla 653, Chile
}

aquiculture to color the flesh of cultured salmonids. Because the characteristic pigmentation is highly desired by consumers, astaxanthin availability has an impact on production costs [8]. Due to its prevalent use in the food, aquiculture, pharmaceutical and cosmetic industries and the increasing demand for natural products, astaxanthin and its sources have great commercial potential $[2,8]$.

Carotenoids are tetraterpenoid compounds that are biosynthesized in the isoprenoid (also known as terpenoid) pathway (Figure 1); the basic units are isopentenyl-pyrophosphate (IPP) and its isomer dimethylallyl-pyrophosphate (DMAPP) [9]. Although an alternate pathway has been described (the deoxyxylulose phosphate, methylerithritol phosphate, or nonmevalonate pathway), IPP is synthesized from acetyl-CoA via the mevalonate (MVA)

\section{Biomed Central}



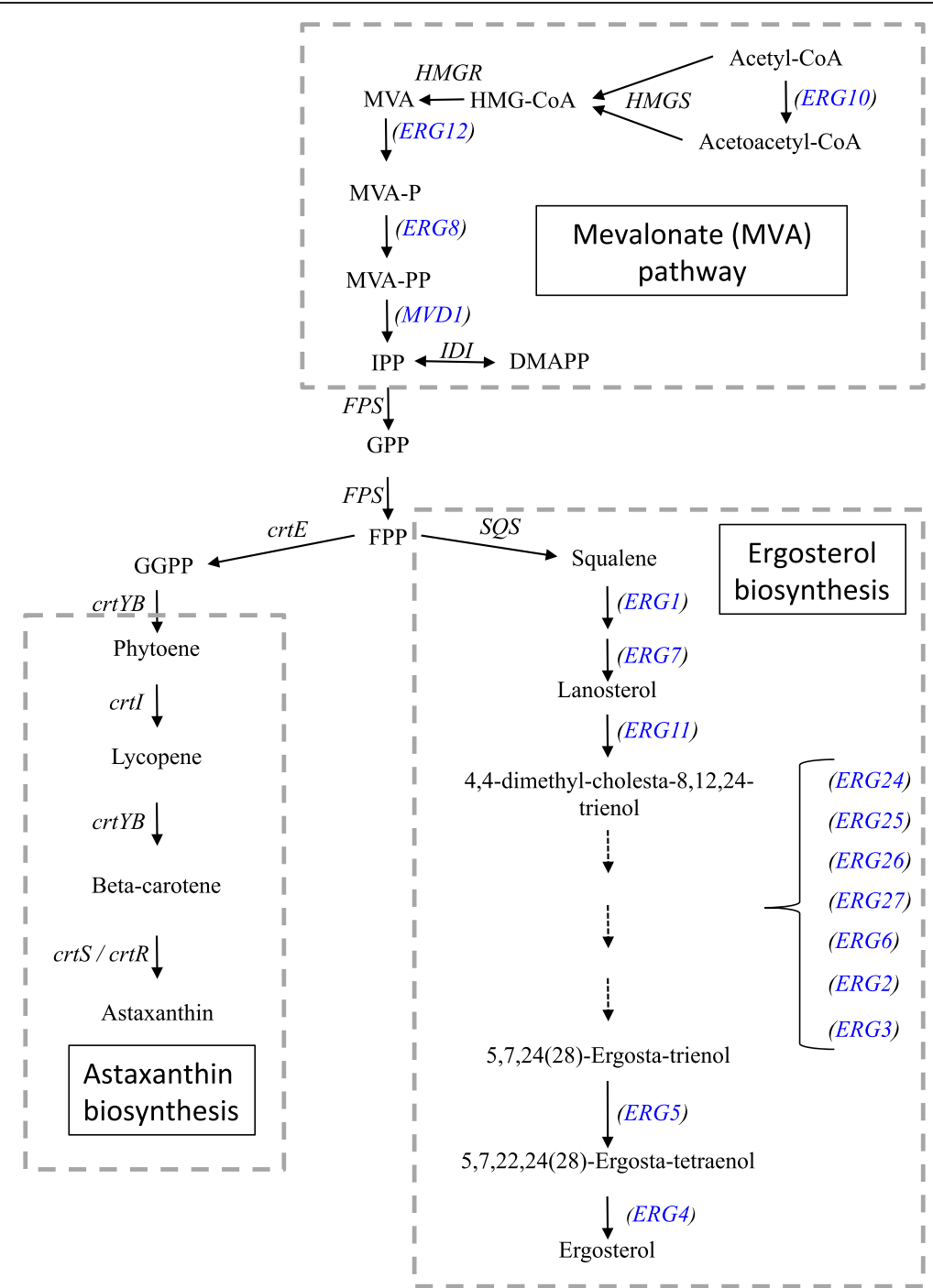

Figure 1 Mevalonate pathway, astaxanthin and ergosterol biosynthesis. The arrows represent the catalytic step with the respective enzymeencoding gene described in X. dendrorhous (gene names without brakets and written in black) and S. cerevisiae (genes between brackets and written in blue). The represented $X$. dendrorhous genes with their Genbank accession number in square brackets are: HMGR [AJ884949], IDI [DQ235686], crtE [DQ012943], crtYB [DQ016503], crtI [Y15007], crtS [EU713462] and crtR [EU884133]. The X. dendrorhous HMGS, FPS and SQS gene sequences are submitted in patents [DI059433.1, DI032788.1 and EA489199, respectively]. The following S. cerevisiae genes are represented: ERG10 [NM_001183842], ERG13 [NM_001182489], ERG12 [AN: NM_001182715], ERG8 [NM_001182727], MVD1 [NM_001183220], ERG20 [NM_001181600], ERG1 [M64994], ERG7 [U23488.1], ERG11 [NM_001179137], ERG24 [NM_001183118], ERG25 [NM_001181189], ERG26 [NM_001180866], ERG27 [NM_001181987], ERG6 [NM_001182363], ERG2 [NM_001182709], ERG3 [NM_001181943], ERG5 [NM_001182511], and ERG4 [NM_001180877]. Abbreviations: 3-hydroxy-3-methylglutaryl-CoA (HMG-CoA), mevalonate (MVA), mevalonate-5-phosphate (MVA-P), mevalonate-5-pyrophosphate (MVA-PP), isopentenyl-pyrophosphate (IPP), dimethylallyl-pyrophosphate (DMAPP), geranyl-pyrophosphate (GPP), farnesyl-pyrophosphate (FPP), geranylgeranyl-pyrophosphate (GGPP).

pathway in most eukaryotes [10]. Five genes control this pathway, and among them, the expression of the gene that encodes hydroxymethylglutaryl-CoA (HMG-CoA) reductase, HMGR, is strongly regulated at different levels (transcription, post-translational and proteolysis) [11]. In the isoprenoid synthesis pathway (Figure 1), DMAPP and IPP are condensed by prenyl transferases to form geranyl-pyrophosphate (GPP), and the addition of a second molecule of IPP gives rise to farnesyl pyrophosphate
(FPP) [9]. Squalene, the precursor of sterols, is formed by the condensation of two molecules of FPP by squalene synthase [12]. For the biosynthesis of carotenoids, a third IPP unit is added to FPP, generating geranylgeranyl-pyrophosphate (GGPP). The condensation of two molecules of GGPP forms the first carotenoid in this biosynthetic pathway, phytoene [13]. During $X$. dendrorhous carotenogenesis, lycopene is formed by four successive desaturations of phytoene; cyclization of the ends of lycopene produces 
beta-carotene [14]. Unlike other astaxanthin-producing organisms, $X$. dendrorhous has a single astaxanthin synthase (encoded by the $\mathrm{crtS}$ gene) that catalyzes the ketolation and hydroxylation of beta-carotene to produce astaxanthin $[15,16]$. This enzyme is related to a $3 \mathrm{~A}$ subfamily member of the cytochrome P450 protein family $[15,16]$. Cytochrome P450 proteins (P450s) are hemecontaining monooxygenases that are present in organisms from all domains of life [17]; P450s have significant roles in the oxidative metabolism of many exogenous and endogenous substrates [18]. In their active state, these enzymes are reduced by electrons that are supplied by NAD $(\mathrm{P}) \mathrm{H}$ through a $\mathrm{P} 450$ redox partner [19], which in eukaryotes is a cytochrome P450 reductase [20]. In X. dendrorhous, the crt $\mathrm{R}$ gene encodes the yeast cytochrome P450 reductase that is essential for the synthesis of astaxanthin [21]. However, the $X$. dendrorhous crtR gene is different from the $c r t R$ gene originally described in cyanobacterium Synechocystis sp., which encodes a betacarotene hydroxylase [22].

Sterols and carotenoids are derived from IPP. Sterols are essential structural and regulatory components of eukaryotic cell membranes, modulating their thickness, fluidity and permeability [23]. Ergosterol is the principal sterol in yeasts, and two cytochrome P450s are involved in its biosynthesis: CYP51 (lanosterol 14-demethylase) and CYP61 (C-22 sterol desaturase), which in Saccharomyces cerevisiae are encoded by the ERG11 and ERG5 genes, respectively [24] (Figure 1). An erg5 S. cerevisiae mutant strain is viable but unable to synthesize ergosterol [25]. Interestingly, one of the major bottlenecks in ergosterol biosynthesis is the reaction catalyzed by HMG-CoA reductase [26]. As MVA is a common precursor in ergosterol and carotenoid biosynthesis, its synthesis should also be an important bottleneck in the biosynthesis of astaxanthin in $X$. dendrorhous. Based on these observations, this study aimed to identify and characterize the $X$. dendrorhous $\mathrm{C}-22$ sterol desaturase encoding gene, CYP61, and to evaluate the effect of its disruption on yeast ergosterol production and carotenogenesis.

\section{Results}

\section{Cloning and sequence analysis of the CYP61 gene from $X$. dendrorhous}

Our X. dendrorhous genomic database was analyzed with the BLAST tool of the CLC Genomics Workbench 5 software using as query several CYP61 gene sequences available in the GenBank database. In this way, we were able to identify a putative CYP61 gene (hereafter CYP61 gene) from $X$. dendrorhous, which allowed us to design specific primers to amplify and clone this gene.

A fragment of approximately 4,200 bp [GenBank: JX183236] was PCR-amplified using genomic DNA from strain UCD 67-385 as a template and the primer set CYP61up2.F + CYP61dw2.R (Table 1). This fragment was inserted at the EcoRV site of the pBluescript SKplasmid, generating pBS-gCyp61. In parallel, the $X$. dendrorhous CYP61 cDNA was screened in a cDNA library by PCR using plasmid DNA from different clone mixtures as templates and the primer pair CYP61.F + CYP61.R (Table 1). The recombinant plasmid pBS-cCyp61, which contained the CYP61 gene cDNA with an ORF of 1,581 bp [GenBank: JX183235], was isolated. The sequence analysis of the genomic and cDNA versions of the CYP61 gene allowed us to determine that this gene consists of nine exons of 156, 152, 114, 75, 81, 441, 169, 320 and 73

\section{Table 1 Primers designed and used in this work}

\begin{tabular}{llll}
\hline $\mathbf{N}^{\mathbf{0}}$ & Primer & Sequence $\mathbf{5}^{\prime}$ to $\mathbf{3}^{\prime}$ & Target \\
\hline 1 & H-out.F & CTCGATGAGCTGATGCTTTG & Hygromycin B resistance cassette \\
2 & H-out.R & TCCATCACAGTTGCCAGTG & Hygromycin B resistance cassette \\
3 & Zeo.F & TGAACAGGGTCACGTCGT & Zeocin resistance cassette \\
5 & Zeo.R & CGCTGATGAACAGGGTCAC & Zeocin resistance cassette \\
6 & CYP61up2.F & CTGGAGCCGAATTCATTGAT & CYP61 gene \\
7 & CYP61dw2.R & AGGAGGCAGAGTGGTTGAGA & CYP61 gene \\
8 & CYP61b.F & GTCGGAGGAAGAGCAGTTG & CYP61 gene \\
9 & CYP61.F & CTGAGCCCTGTCTTGTTGCC & CYP61 gene \\
RT-qPCR (The pairs of primers used had efficiency greater than 95\%, as determined by standard curves with a correlation coefficient of R2 $\geq 0.996):$ \\
10 & mactF-RT & CCGCCCTCGTGATTGATAAC & ACT gene \\
11 & mactR-RT & TCACCAACGTAGGAGTCCTT & ACT gene \\
12 & hmgR.F-RT & GGCCGATCGCTATACATCCGTT & HMGR gene \\
13 & hmgR.R-RT & ATCCAGTGATGGCAGAAGGCT & HMGR gene \\
\hline
\end{tabular}

$\mathrm{F}$ and $\mathrm{R}$ in the primer name indicate the primer orientation. 


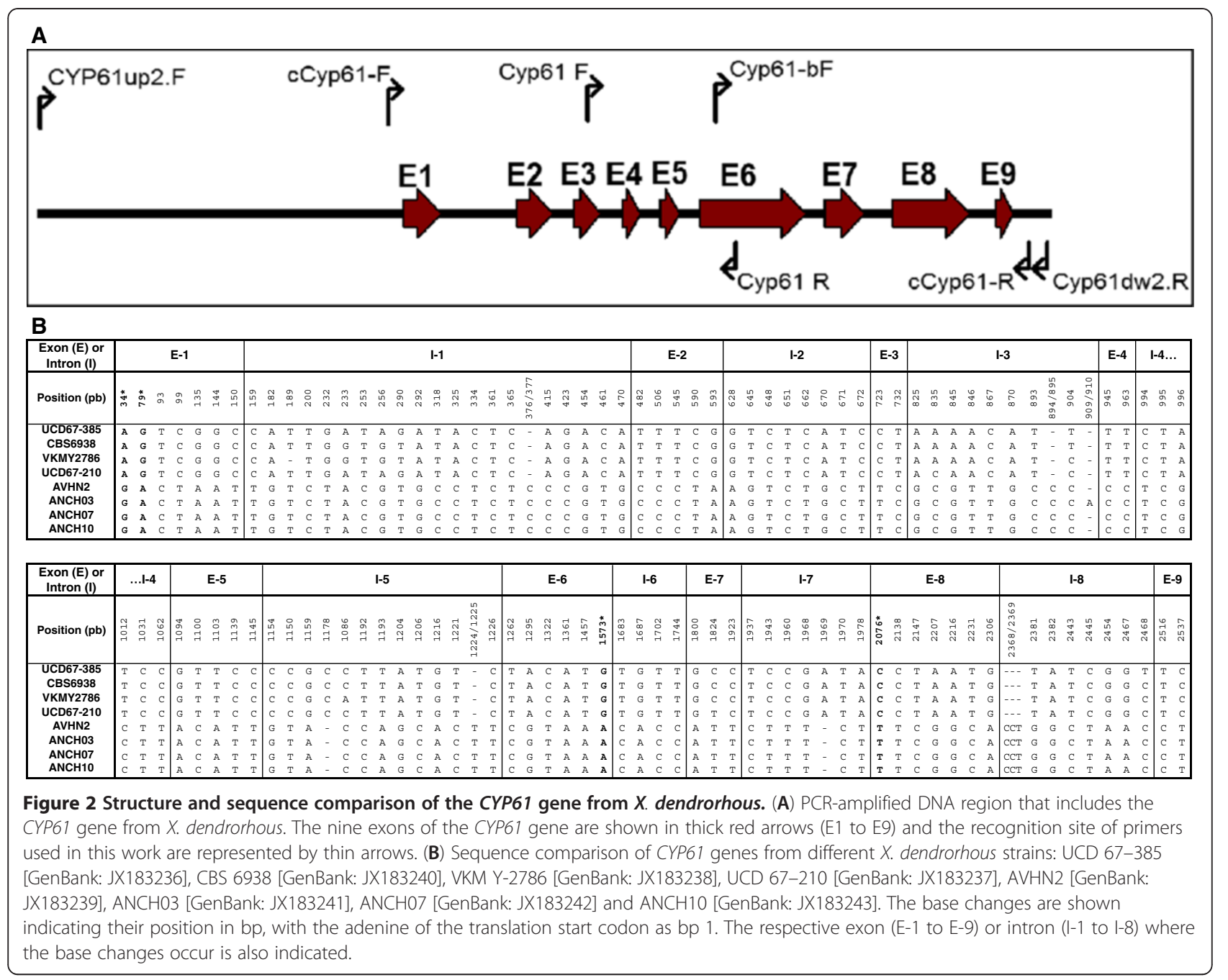

bp, and eight introns of 317, 82, 90, 83, 84, 79, 116 and 111 bp (Figure 2A). The CYP61 gene encodes a putative 526 amino acid CYP61 protein with a predicted molecular weight of $59.6 \mathrm{kDa}$ and $\mathrm{p} I$ of 6.48 . The CYP61 deduced protein from $X$. dendrorhous shares $43 \%$ identity and $65 \%$ similarity at $95 \%$ sequence coverage with the Saccharomyces cerevisiae C22-sterol desaturase (CYP61, Swiss-Prot: P54781.1). This protein belongs to the cytochrome P450 protein family and is involved in the second last step of the ergosterol biosynthesis, the conversion of 5,7,24(28)-ergostatrienol into 5,7,22,24(28)ergostatetraenol [25].

The CYP61 gene from several $X$. dendrorhous strains (VKM Y-2786, CBS 6938, UCD 67-210, ANCH03, ANCH07, ANCH10 and AVHN2 (Table 2) was PCRamplified using $P f u$ DNA pol, and each amplicon was sequenced [GenBank: JX183238, JX183240, JX183237, JX183241, JX183242, JX183243 and JX183239, respectively]. We found several base changes, but most of them were located in the intronic regions. Only four base changes produced amino acid replacements; the adenine, guanine, guanine and cytosine at positions $34,79,1,573$ and 2,075 were converted to guanine, adenine, adenine and thymine (numeration according to the CYP61 gene translation start in strain UCD 67-385), resulting in T12A, A27T, R306K and P409S variations at the deduced amino acid sequence, respectively (Figure $2 \mathrm{~B}$ ).

Even though the amino acid sequences are extremely diverse among the cytochrome $\mathrm{P} 450$ protein family, their structural fold is highly conserved [27]. Several cytochrome P450 secondary structural elements in the deduced CYP61 protein from $X$. dendrorhous were predicted with the CYP450 Engineering database [28] (Figure 3). This included alpha helices A, B, C, D, F, G, H, I, J, K, K' and $\mathrm{L}$, beta-sheets $1-1,1-2,1-5,3-1,1-4,2-1,2-2,1-3$, $3-3,4-1,4-2$ and $3-2$, the meander loop, which may be involved in the stabilization of the tertiary structure and heme binding, and the Cys pocket that contains the 
Table 2 Strains and Plasmids used and built in this work

Strains:

E. coli:

$\mathrm{DH}-5 \mathrm{a}$

F- 80d lacZ $\Delta$ M15 $\Delta$ (lacZY-argF) U169 deoR recA1 endA1 hsdR17(rk- mk+) phoA

X. dendrorhous: supE44l- thi-1 gyrA96 relA1

UCD 67-385

ATCC

Diploid strain [30]

$385-\operatorname{cyp} 61^{(+/-)}$

(385-CYP61/cyp6 $1^{\text {hph }}$ ). Heterozygote transformant derived from UCD 67-385 containing

This work an allele of the CYP61 locus interrupted with a hygromycin B resistance cassette.

$385-\operatorname{cyp} 61^{(-/-)}$

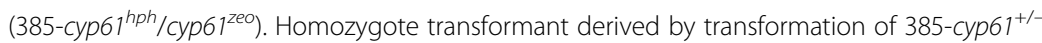
with both CYP61 alleles interrupted, one with a hygromycin B resistance cassette and the other with a zeocin resistance cassette.

CBS 6938

ATCC 96594, wild type.

ATCC

$\left(\right.$ CBS-cyp61 $\left.{ }^{\text {hph }}\right)$. Hemizygote transformant derived from CBS 6938. The single CYP61 locus was interrupted with a hygromycin B resistance cassette.

Chilean native isolate, wild type.

(Av2-cyp61 $1^{\text {zeo }}$ ). Hemizygote transformant derived from AVHN2. The single CYP61 locus was interrupted with a zeocin resistance cassette.

ATCC 24202, wild type (Phaffia rhodozyma)

This work

This work

$\mathrm{AVHN2}^{*}$

Av2-cyp $61^{(-)}$

UCD 67-210

Wild-type strain.

VKM Y-2786

Chilean Antarctic native isolate, wild type.

Chilean Antarctic native isolate, wild type.

Chilean Antarctic native isolate, wild type.

Our Lab collection

This work

ATCC

VKM

Our Lab collection

Our Lab collection

Our Lab collection

Stratagene

[31]

$\mathrm{pBS}$ containing at the EcoRV site a cassette of $1.8 \mathrm{~kb}$ bearing the E. coli-Hygromycin B resistance (hph) gene under EF-1 a promoter and GPD transcription terminator of $X$. dendrorhous.

pIR-zeo

pBS containing at the EcoRV site a cassette of $1.2 \mathrm{~kb}$ bearing the Streptoalloteichus hindustanus Zeocin resistance Sh ble gene under EF-1 a promoter and GPD transcription terminator of $X$. dendrorhous.

pBS-gCyp61

pBS containing at the EcoRV site a 4,224 bp DNA fragment containing the $X$. dendrorhous CYP61 gene amplified by PCR with primers CYP61up2.F and CYP61dw2.R.

pBS-cyp61/Hyg

pBS-gCyp61 bearing the Hygromycin B resistance cassette at the EcoRV site that interrupts the CYP61 gene.

pBS-cyp61/Zeo

pBS-gCyp61 bearing the Zeocin resistance cassette at the EcoRV site that interrupts the CYP61 gene.

pBS bearing the CDNA of the CYP61 gene. The CDNA measures 1,752 bp with an ORF of 1,581 bp.
This work

This work

This work

This work

This work
pBS-cCyp61

${ }^{*}: X$. dendrorhous Chilean native isolates confirmed by ITS, D1/D2 and IGS regions sequences. The following abbreviations are used for microorganism culture collections: CBS, Centraalbureau voor Schimmelcultures, Utrecht, Netherlands; ATCC, American Type Culture Collection, Manassas, USA; UCD, Phaff Yeast Culture Collection, Department of Food Science and Technology, University of California at Davis, Davis, USA; VKM, The All-Russian Collection of Microorganisms, Moscow, Russia.

conserved cysteine involved in heme binding. There are three totally conserved amino acids in the cytochrome P450 protein family, the glutamic acid and arginine of the E-X-X-R motif at the K-helix, which are involved in stabilizing the core and heme binding, and the heme binding cysteine [28], and these residues are present in the predicted CYP61 protein. Additionally, we were able to predict the putative hydrophobic transmembrane segment at the CYP61 amino terminus, which could anchor the protein to the endoplasmic reticulum [29].

\section{The CYP61 gene mutation}

To study the function of the CYP61 gene in X. dendrorhous, mutant cyp61- strains were generated. The wild-type strains UCD 67-385 and CBS 6938 were transformed with plasmid pBS-cyp61/Hyg, and strain AVHN2 was transformed with plasmid pBS-cyp61/Zeo. All transformations were performed with linearized plasmids as indicated in Figure 4. Through a double homologous recombination event, the donor DNA fragment containing the CYP61 gene interrupted by one of the 


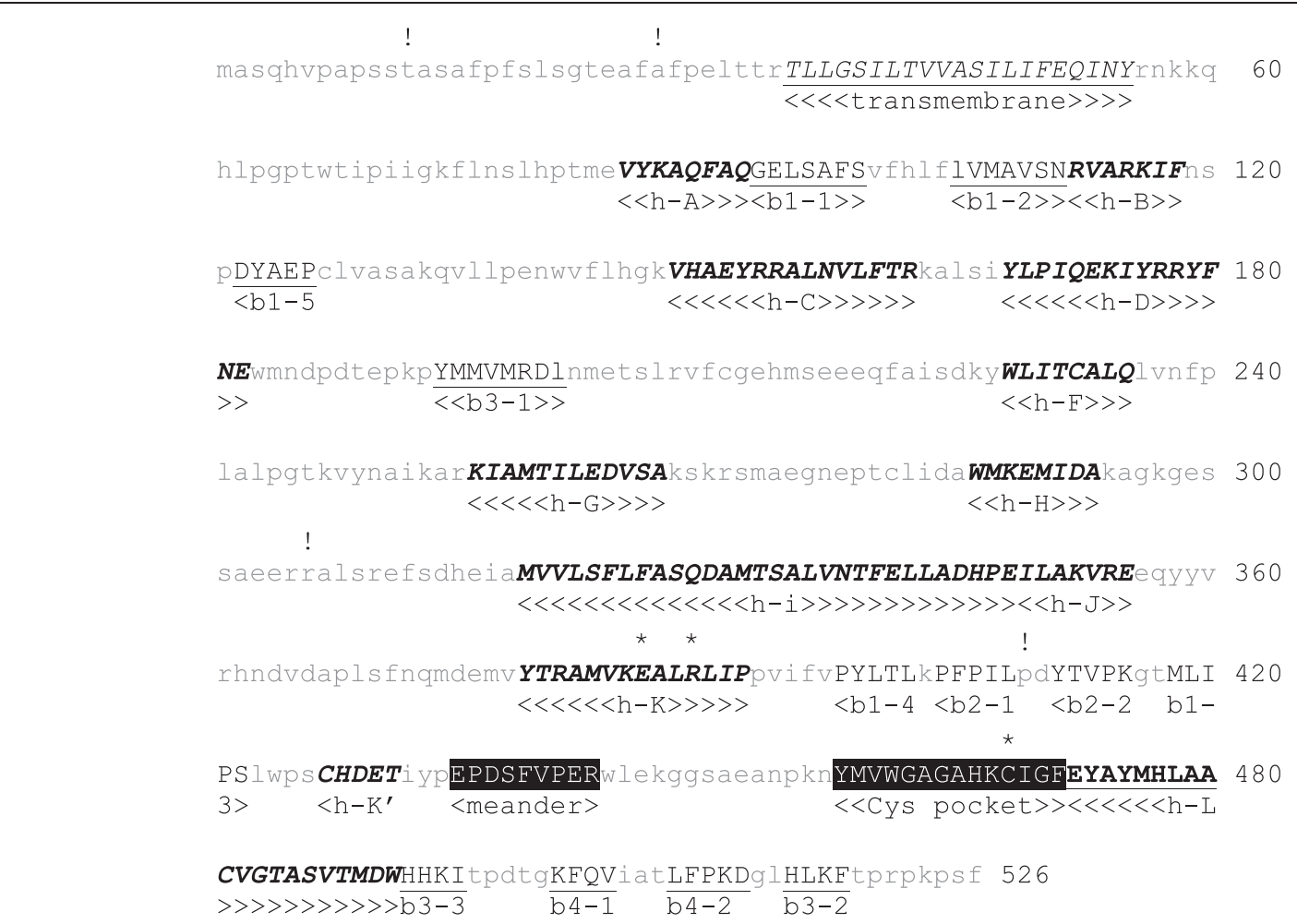

Figure 3 Deduced $\boldsymbol{X}$. dendrorhous CYP61. Secondary structural elements were predicted with the CYP450 Engineering database, and the potential transmembrane region was predicted with TMpred. Structural elements are in capital letters with the name of the corresponding feature underneath them. Underlined and in italics: possible transmembrane helix. In bold and italics: alpha helices. Underlined: Beta-sheets. In white letters and highlighted in black: meander loop and Cys pocket. The asterisks $\left(^{*}\right)$ indicate the three totally conserved amino acids among cytochromes P450, and the exclamation points (!) show the amino acid variation found in the deduced CYP61 from different X. dendrorhous strains.

two resistance markers replaced the CYP61 gene in the yeast chromosome. In this way, we obtained the transformant strains 385-cyp61 $1^{\text {hph }}$, CBS-cyp61 $1^{\text {hph }}$ and Av2cyp61 $1^{z e o}$ (Table 2). The genotype modifications in the transformant strains were validated by PCR reactions using specific primers for the CYP61 gene, zeocin or hygromycin B resistance cassettes (Table 1) and genomic DNA from the parental and transformant strains. The amplicons confirmed the CYP61 gene interruption (Figure 5). However, as strain UCD 67-385 is diploid [30] and we were able to detect a CYP61 wild-type allele, the resulting strain $385-c y p 61^{h p h}$ is heterozygous (385-CYP61/ cyp61 $\left.{ }^{h p h}\right)$. For this reason, strain 385-CYP61/cyp61 ${ }^{h p h}$ was transformed with the linearized plasmid pBS-cyp61/Zeo obtaining the cyp61- homozygote mutant strain 385cyp $61^{h p h} /$ cyp $61^{z e o}$ (Figure 5). The ploidy levels of strains CBS 6938 and AVHN2 are unknown; based on random mutagenesis experiments and by transformation of carotenogenic genes performed at our laboratory [21,31], we estimate that these strains are aneuploid. In these cases, the PCR-based genotype analysis determined that a unique CYP61 gene copy was mutated in strains CBS-cyp61 $1^{\text {hph }}$ and Av2-cyp61 $1^{z e o}$ (Figure 5), indicating that these strains are hemizygous, so a second transformation event was not necessary in these mutants. Interestingly, a clear difference in the color phenotype could be distinguished among all the cyp $61^{-}$mutants and their corresponding parental strains, indicating alterations in carotenoid biosynthesis (see below).

CYP61 gene mutant phenotype evaluation: ergosterol and carotenoid production

To analyze and compare the cyp61- mutant phenotypes, the seven strains UCD 67-385, 385-CYP61/cyp61 ${ }^{h p h}$, 385-cyp61 $1^{h p h} /$ cyp61 $61^{z e o}$, CBS 6938, CBS-cyp61 $1^{h p h}$, AVHN2 and Av2-cyp61 $1^{z e o}$ were cultivated in YM complete medium for 5 days at $22^{\circ} \mathrm{C}$ with constant agitation. Growth was measured by the culture absorbance at $600 \mathrm{~nm}$, and samples were taken after 24, 72 and $120 \mathrm{~h}$ of cultivation. The samples were processed to determine the yeast dry weight and to extract sterols, carotenoids and RNA as described in the Materials and Methods section.

As in other species, the CYP61 gene is involved in the ergosterol biosynthesis, so we evaluated the sterol production and composition in the cyp $61^{-}$mutants by RPHPLC. Figure 6 shows representative chromatograms obtained from sterols extracted from strains UCD 67385 and $385-c y p 61^{h p h} / c y p 61^{z e o}$, representing the parental 


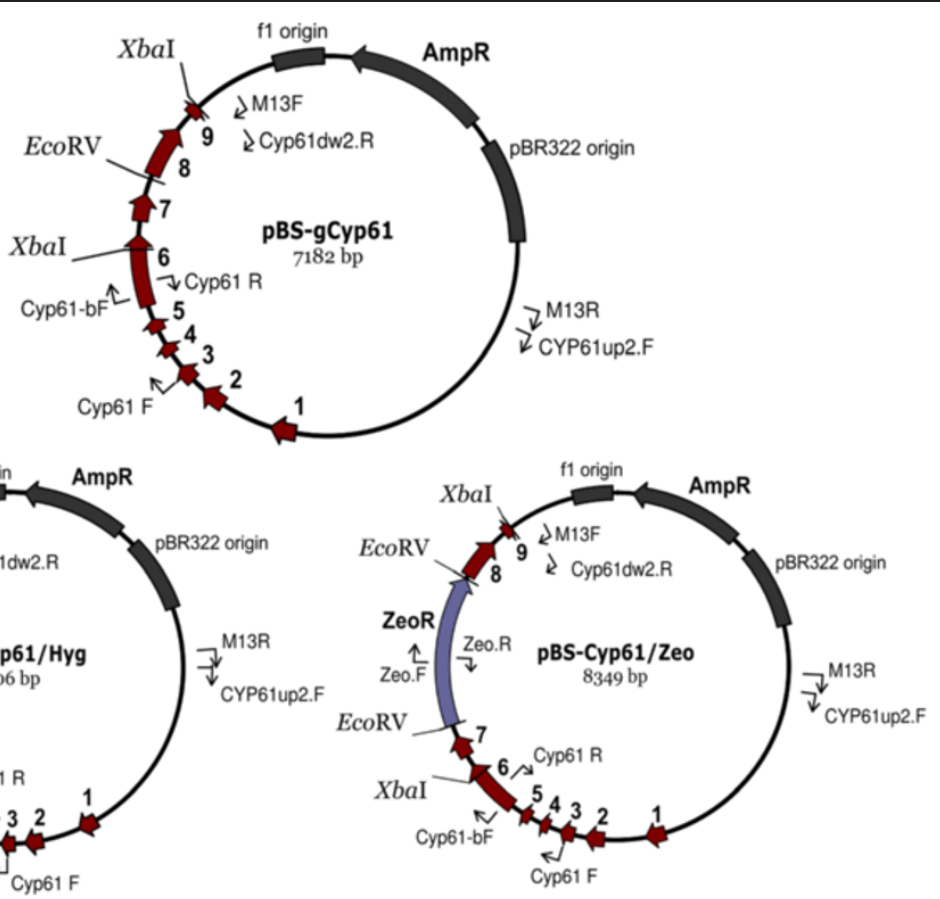

Figure 4 Plasmids constructed in this work. In each plasmid illustration, relevant features for this work, such as endonuclease recognition sites and primer binding sites (thin arrows), are shown. Some elements of the original plasmid (pBluescript SK-) were kept and shown in gray. Plasmid pBS-gCyp61 harbors the genomic version of the CYP61 gene from $X$. dendrorhous that was PCR-amplified with primers CYP61up2.F and Cyp61dw2.R. Red thick arrows along with a number represent the nine exons of the CYP61 gene. Plasmids pBS-Cyp61/Hyg and pBS-Cyp61/Zeo were built by inserting the hygromycin B (HygR, in green) and zeocin (ZeoR, in violet) resistance expression cassettes, respectively, at the EcoRV site of plasmid pBS-gCyp61. To linearize the plasmids for transformation purposes, pBS-Cyp61/Hyg and pBS-Cyp61/Zeo were digested with Xbal.

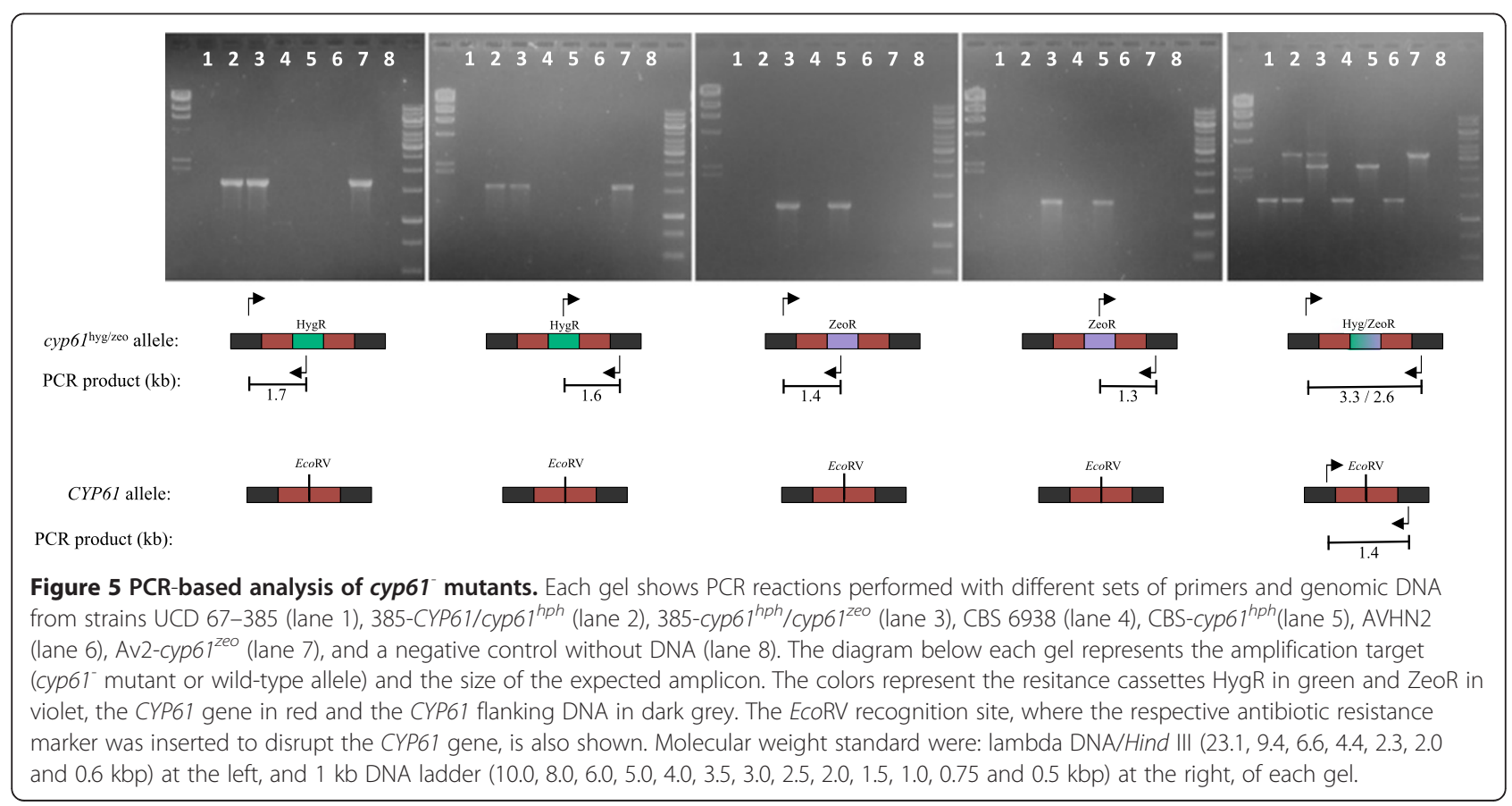



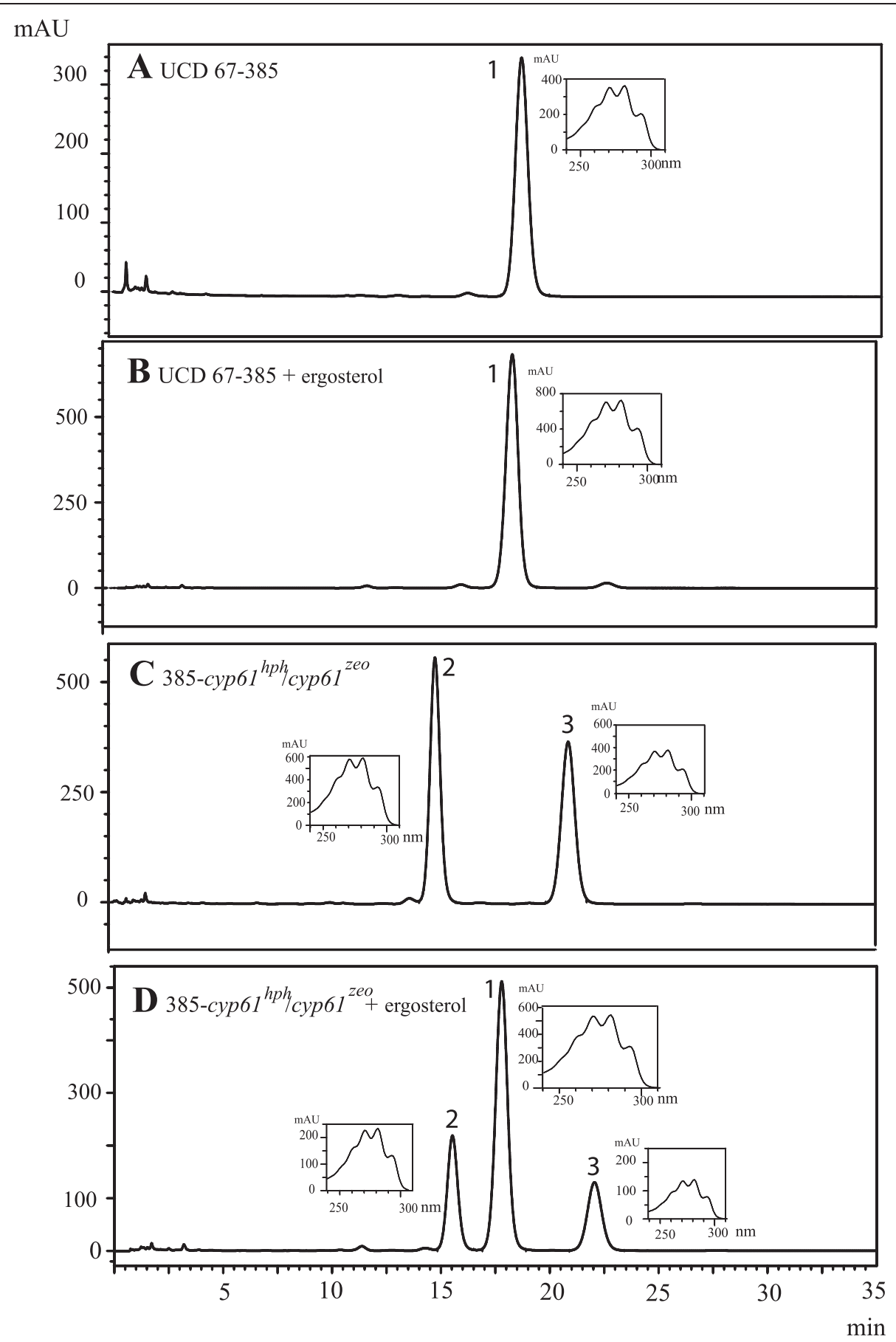

Figure 6 RP-HPLC sterols analysis from UCD 67-385 and 385-cyp61 ${ }^{\text {hph }} /$ cyp61 ${ }^{\text {zeo }}$ strains. Chromatograms (at $280 \mathrm{~nm}$ ) correspond to sterols extracted from strains as described in the Materials and Methods section. Beside each peak (peaks № 1 to 3), the corresponding spectra were included. Sterols were analyzed from UCD 67-385 wild-type (A), UCD 67-385 wild-type co-injected with standard ergosterol (B), 385-cyp61 $1^{\text {hph }}$ / cyp6 $1^{\text {zeo }}$ mutant (C) and 385-cyp61 hph/cyp6 $7^{\text {zeo }}$ co-injected with standard ergosterol. Ergosterol corresponds to peak № 1.

and the cyp $61^{-}$mutant strains, respectively. In wild-type strains, we observed a predominant peak (peak 1) at the $280 \mathrm{~nm}$ channel at approximately $18 \mathrm{~min}$ with the ergosterol characteristic spectra (Figure 6 A), and its identity was confirmed by co-injecting each sample with standard ergosterol (Figure 6 B). On the other hand, in the analysis of the sterols from the homozygous and hemizygous cyp $61^{-}$mutants, two peaks were observed with retention times close to 15 (peak 2) and $21 \mathrm{~min}$ (peak 3) (Figure $6 \mathrm{C}$, Table 3). These two peaks did also 
Table 3 Sterol composition according to their RP-HPLC profile of wild-type and cyp61 X. dendrorhous mutant strains (in $\mathbf{m g} / \mathrm{g}$ dry yeast weight)

\begin{tabular}{|c|c|c|c|c|c|c|c|c|c|}
\hline \multirow[b]{3}{*}{ Cultivation time (h) } & \multicolumn{9}{|c|}{ Strains } \\
\hline & \multicolumn{3}{|c|}{ UCD 67-385 } & \multicolumn{3}{|c|}{$385-c y p 61^{(+/-)}$} & \multicolumn{3}{|c|}{ 385-сур61 $1^{(-/-)}$} \\
\hline & 24 & 72 & 120 & 24 & 72 & 120 & 24 & 72 & 120 \\
\hline Ergosterol* $^{*}$ & $4.74 \pm 0.53$ & $3.10 \pm 0.09$ & $2.24 \pm 0.42$ & $3.19 \pm 0.48$ & $2.87 \pm 0.32$ & $2.91 \pm 0.34$ & ND & ND & ND \\
\hline Peak $2^{* *}$ & $0.23 \pm 0.03$ & $0.030 \pm 0.003$ & $0.10 \pm 0.05$ & $0.62 \pm 0.05$ & $0.11 \pm 0.03$ & $0.12 \pm 0.02$ & $6.34 \pm 2.68$ & $2.36 \pm 0.74$ & $2.39 \pm 0.27$ \\
\hline Peak $3^{* * *}$ & $0.19 \pm 0.04$ & ND & $0.09 \pm 0.02$ & $0.11 \pm 0.01$ & $0.02 \pm 0.01$ & $0.01 \pm 0.003$ & $1.65 \pm 0.84$ & $1.91 \pm 0.51$ & $2.20 \pm 0.42$ \\
\hline \multirow[t]{3}{*}{ Total Sterols } & $5.16 \pm 0.57$ & $3.13 \pm 0.09$ & $2.40 \pm 0.49$ & $3.96 \pm 0.44$ & $2.99 \pm 0.35$ & $3.04 \pm 0.36$ & $8.14 \pm 3.42$ & $4.27 \pm 1.24$ & $4.59 \pm 0.70$ \\
\hline & \multicolumn{6}{|c|}{ Strains } & & & \\
\hline & \multicolumn{3}{|c|}{ CBS 6938} & \multicolumn{3}{|c|}{ CBS-cyp61 $1^{(-)}$} & & & \\
\hline Cultivation time (h) & 24 & 72 & 120 & 24 & 72 & 120 & & & \\
\hline Ergosterol* $^{*}$ & $3.31 \pm 0.60$ & $2.39 \pm 0.56$ & $2.37 \pm 0.11$ & ND & ND & ND & & & \\
\hline Peak $2^{* *}$ & $0.07 \pm 0.04$ & $0.06 \pm 0.02$ & $0.06 \pm 0.01$ & $2.00 \pm 0.34$ & $1.24 \pm 0.02$ & $1.23 \pm 0.04$ & & & \\
\hline Peak $3^{* * *}$ & $0.03 \pm 0.001$ & $0.02 \pm 0.01$ & $0.03 \pm 0.01$ & $2.38 \pm 0.29$ & $2.60 \pm 0.08$ & $3.05 \pm 0.17$ & & & \\
\hline \multirow[t]{3}{*}{ Total Sterols } & $3.45 \pm 0.56$ & $2.41 \pm 0.59$ & $2.46 \pm 0.11$ & $4.38 \pm 0.61$ & $3.85 \pm 0.1$ & $4.28 \pm 0.21$ & & & \\
\hline & \multicolumn{6}{|c|}{ Strains } & & & \\
\hline & \multicolumn{3}{|c|}{ AVHN2 } & \multicolumn{3}{|c|}{ AV2-cyp61 $1^{(-)}$} & & & \\
\hline Cultivation time (h) & 24 & 72 & 120 & 24 & 72 & 120 & & & \\
\hline Ergosterol* $^{*}$ & $1.59 \pm 0.62$ & $2.35 \pm 0.59$ & $3.27 \pm 0.38$ & ND & ND & ND & & & \\
\hline Peak $2^{* *}$ & ND & $0.04 \pm 0.01$ & $0.04 \pm 0.01$ & $1.68 \pm 0.78$ & $2.10 \pm 0.32$ & $1.78 \pm 0.13$ & & & \\
\hline Peak $3^{* * *}$ & ND & ND & ND & $1.39 \pm 0.82$ & $2.27 \pm 0.18$ & $2.39 \pm 0.52$ & & & \\
\hline Total Sterols & $1.59 \pm 0.62$ & $2.39 \pm 0.59$ & $3.31 \pm 0.39$ & $3.16 \pm 1.70$ & $4.36 \pm 0.49$ & $4.11 \pm 0.64$ & & & \\
\hline
\end{tabular}

Table shows the mean values \pm standard deviations of three independent experiments. Retention time: *: 18 min; **: 15 min; ***: 22 min. ND: Not detected.

have the sterols characteristic spectra, but their retention time was different than that of ergosterol. This last observation was also confirmed by co-injecting the extracted sterols with standard ergosterol, resulting in three peaks at approximately 15, 18 and $22 \mathrm{~min}$ (Figure $6 \mathrm{D}$ ). Additionally, the identity of the sterols was determined by GC-MS (Additional file 1: Figure S1), confirming that wild-type strains produced mainly ergosterol and that the mutants instead accumulated ergosta-5,8,22-trien-3-ol and ergosta-5,8-dien-3-ol. Considering the relative abundance of each sterol obtained by GC-MS and RP-HPLC, peaks 2 and 3 in the RP-HPLC chromatogram from the cyp $61^{-} \mathrm{mu}-$ tant strain (Figure $6 \mathrm{C}$ ) should correspond to ergosta-5,8dien-3-ol and ergosta-5,8,22-trien-3-ol, respectively.

Table 3 summarizes the sterol composition of the seven strains at different cultivation times. In general, when compared to the corresponding parental strain, the total sterol content was greater in the cyp61- mutants. In addition, the sterols produced by the cyp $61^{-}$mutant strains corresponding to peaks 2 and 3 were at ratios of $55 \%$ and $44 \%, 32 \%$ and $68 \%, 48 \%$ and $52 \%$ in the $385-c y p 61^{\text {hph }} /$ cyp61 $1^{z e o}$, CBS-cyp61 $1^{\text {hph }}$, Av2-cyp61 $1^{z e o}$ strains, respectively. In the heterozygous strain 385-CYP61/cyp $61^{h p h}$, the main sterol produced was ergosterol.

A visible change in the color of the cyp $61^{-}$mutants was evident when compared to their corresponding parental

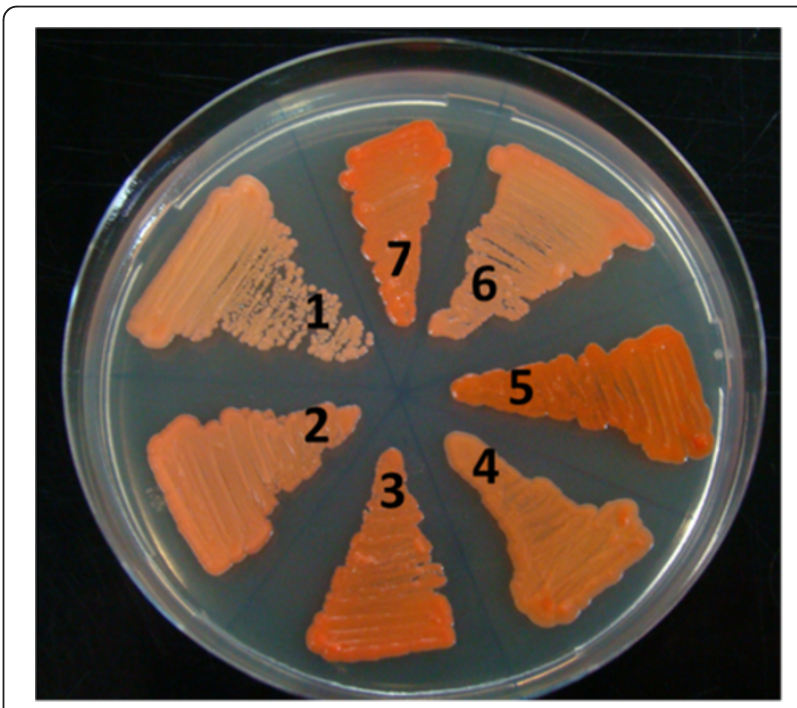

Figure 7 Color phenotype of cyp61 mutant and wild-type strains. Cultures in solid YM complete media of strains UCD 67-385 (1), 385-CYP61/cyp61 $1^{\text {hph }}$ (2), 385-cyp61 hph /cyp61 zeo (3), AVHN2 (4), Av2-cyp61 $1^{\text {zeo }}$ (5), CBS 6938 (6) and CBS-cyp61 ${ }^{\text {hph }}$ (7). 
Table 4 Carotenoid composition of wild-type and cyp61 X. dendrorhous mutant strain (in ppm)

\begin{tabular}{|c|c|c|c|c|c|c|c|c|c|}
\hline \multirow[b]{3}{*}{ Cultivation time (h) } & \multicolumn{9}{|c|}{ Strains } \\
\hline & \multicolumn{3}{|c|}{ UCD 67-385 } & \multicolumn{3}{|c|}{$385-c y p 61^{(+/-)}$} & \multicolumn{3}{|c|}{ 385-сyp61 ${ }^{(-/-)}$} \\
\hline & 24 & 72 & 120 & 24 & 72 & 120 & 24 & 72 & 120 \\
\hline Astaxanthin & $52.6 \pm 22.3$ & $26.3 \pm 2.7$ & $224.0 \pm 42.1$ & $89.1 \pm 13.4$ & $34.9 \pm 5.1$ & $223.7 \pm 8.6$ & $126.5 \pm 31.0$ & $49.8 \pm 18.2$ & $434.7 \pm 56.2$ \\
\hline Phoenicoxanthin & ND & ND & ND & ND & ND & ND & ND & ND & ND \\
\hline Cantaxanthin & ND & ND & $13.4 \pm 3.3$ & ND & ND & ND & ND & ND & ND \\
\hline HO-keto- $\gamma$-carotene & ND & $1.0 \pm 0.5$ & ND & ND & $1.9 \pm 0.3$ & ND & ND & $2.2 \pm 1.3$ & ND \\
\hline HO-keto-torulene & $2.6 \pm 1.1$ & $1.1 \pm 0.2$ & $30.1 \pm 6.7$ & ND & ND & $35.5 \pm 1.0$ & ND & ND & $62.1 \pm 7.3$ \\
\hline Keto- $\gamma$-carotene & $8.0 \pm 4.9$ & $2.7 \pm 1.4$ & $7.8 \pm 1.9$ & ND & $1.2 \pm 0.6$ & $9.7 \pm 1.0$ & ND & $5.7 \pm 2.9$ & $21.4 \pm 7.9$ \\
\hline HO-echinenone & $1.8 \pm 0.6$ & $1.2 \pm 0.9$ & $2.6 \pm 0.5$ & ND & $2.6 \pm 0.5$ & $9.2 \pm 0.4$ & ND & $3.6 \pm 1.6$ & $15.6 \pm 4.4$ \\
\hline Echinenone & ND & ND & $2.0 \pm 0.4$ & ND & ND & ND & ND & ND & ND \\
\hline Lycopene & $4.0 \pm 2.0$ & ND & ND & ND & $1.4 \pm 0.7$ & $1.1 \pm 1.0$ & ND & $4.3 \pm 1.9$ & ND \\
\hline$\gamma$-carotene & ND & $0.2 \pm 0.03$ & $2.7 \pm 0.5$ & ND & ND & ND & ND & $0.8 \pm 0.4$ & ND \\
\hline$\beta$-carotene & $1.1 \pm 0.5$ & $0.8 \pm 0.3$ & $2.7 \pm 1.1$ & ND & $1.7 \pm 1.0$ & $6.3 \pm 0.8$ & ND & $4.8 \pm 3.5$ & $15.8 \pm 9.1$ \\
\hline \multirow[t]{3}{*}{ Total carotenoids } & $70.7 \pm 26.9$ & $36.1 \pm 8.6$ & $290.1 \pm 53.4$ & $89.1 \pm 13.4$ & $47.6 \pm 7.1$ & $293.7 \pm 9.1$ & $126.5 \pm 31.0$ & $78.2 \pm 26.2$ & $555.1 \pm 75.2$ \\
\hline & \multicolumn{6}{|c|}{ Strains } & & & \\
\hline & \multicolumn{3}{|c|}{ CBS 6938} & \multicolumn{3}{|c|}{ CBS-cyp61 $1^{(-)}$} & & & \\
\hline Cultivation time $(\mathbf{h})$ & 24 & 72 & 120 & 24 & 72 & 120 & & & \\
\hline Astaxanthin & $32.1 \pm 11.2$ & $202.0 \pm 17.7$ & $324.2 \pm 6.7$ & $62.8 \pm 5.4$ & $313.5 \pm 24.1$ & $429.3 \pm 26.5$ & & & \\
\hline Phoenicoxanthin & $13.7 \pm 8.0$ & $8.1 \pm 2.1$ & ND & ND & ND & ND & & & \\
\hline Cantaxanthin & ND & ND & ND & ND & ND & ND & & & \\
\hline HO-keto- $\gamma$-carotene & $2.9 \pm 1.4$ & $9.5 \pm 0.6$ & ND & $2.7 \pm 2.0$ & ND & $12.2 \pm 10.5$ & & & \\
\hline HO-keto-torulene & ND & $20.1 \pm 3.6$ & $25.6 \pm 12.4$ & ND & $76.4 \pm 8.3$ & $72.8 \pm 18.0$ & & & \\
\hline Keto- $\gamma$-carotene & $9.8 \pm 4.6$ & $32.8 \pm 4.6$ & $29.8 \pm 0.45$ & $7.1 \pm 0.8$ & $50.2 \pm 3.5$ & $33.0 \pm 2.97$ & & & \\
\hline HO-echinenone & $1.4 \pm 0.8$ & $21.9 \pm 5.2$ & $15.7 \pm 0.6$ & $3.9 \pm 0.1$ & $24.1 \pm 1.6$ & $18.8 \pm 1.0$ & & & \\
\hline Echinenone & ND & ND & ND & ND & ND & ND & & & \\
\hline Lycopene & $16.0 \pm 1.3$ & ND & ND & $11.9 \pm 4.9$ & $3.2 \pm 0.5$ & $2.9 \pm 0.1$ & & & \\
\hline$\gamma$-carotene & $2.4 \pm 2.0$ & $7.3 \pm 1.6$ & $7.6 \pm 0.5$ & ND & $8.8 \pm 0.2$ & $15.3 \pm 1.7$ & & & \\
\hline$\beta$-carotene & $0.4 \pm 0.2$ & $33.2 \pm 6.8$ & $20.4 \pm 0.7$ & $1.8 \pm 1.2$ & $41.8 \pm 4.2$ & $31.2 \pm 1.4$ & & & \\
\hline \multirow[t]{3}{*}{ Total carotenoids } & $78.9 \pm 21.3$ & $347.2 \pm 36.9$ & $453 \pm 11.1$ & $91.9 \pm 7.44$ & $530.3 \pm 21.4$ & $625.8 \pm 22.9$ & & & \\
\hline & \multicolumn{6}{|c|}{ Strains } & & & \\
\hline & \multicolumn{3}{|c|}{ AVHN2 } & \multicolumn{3}{|c|}{ AV2-cyp61 $1^{(-)}$} & & & \\
\hline Cultivation time (h) & 24 & 72 & 120 & 24 & 72 & 120 & & & \\
\hline Astaxanthin & $15.2 \pm 0.8$ & $116.5 \pm 7.0$ & $131.8 \pm 20.6$ & $16.3 \pm 6.1$ & $118.0 \pm 59.2$ & $143.0 \pm 64.8$ & & & \\
\hline Phoenicoxanthin & ND & ND & ND & ND & ND & ND & & & \\
\hline Cantaxanthin & ND & ND & ND & ND & ND & ND & & & \\
\hline HO-keto- $\gamma$-carotene & ND & $20.0 \pm 1.2$ & $17.9 \pm 2.8$ & ND & $25.3 \pm 7.8$ & $36.8 \pm 16.7$ & & & \\
\hline HO-keto-torulene & $0.7 \pm 0.4$ & $27.0 \pm 10.4$ & $21.1 \pm 2.6$ & $1.1 \pm 0.9$ & $62.8 \pm 22.3$ & $40.6 \pm 9.9$ & & & \\
\hline Keto- $\gamma$-carotene & $3.0 \pm 1.07$ & ND & ND & $1.7 \pm 0.7$ & $13.1 \pm 9.25$ & ND & & & \\
\hline HO-echinenone & $2.1 \pm 0.6$ & $10.9 \pm 5.7$ & $9.9 \pm 0.9$ & ND & $9.3 \pm 7.3$ & $13.6 \pm 2.6$ & & & \\
\hline Echinenone & ND & ND & ND & ND & ND & ND & & & \\
\hline Lycopene & $1.4 \pm 1.0$ & ND & ND & ND & $4.0 \pm 2.5$ & ND & & & \\
\hline$\gamma$-carotene & ND & $0.8 \pm 0.1$ & ND & ND & $2.2 \pm 1.7$ & $1.1 \pm 0.9$ & & & \\
\hline$\beta$-carotene & $1.0 \pm 0.5$ & $19.7 \pm 12.0$ & $12.0 \pm 2.9$ & $1.9 \pm 0.9$ & $25.4 \pm 7.6$ & $20.4 \pm 4.7$ & & & \\
\hline Total carotenoids & $24.9 \pm 2.8$ & $195.3 \pm 33.7$ & $193.4 \pm 19.0$ & $25.0 \pm 6.9$ & $274.6 \pm 24.1$ & $258.6 \pm 76.7$ & & & \\
\hline
\end{tabular}

Table shows the mean values \pm standard deviations of three independent experiments. ND: Not detected. 
strain (Figure 7). The first ones had a more intense red color, suggesting that the mutant strains produced more carotenoids. This observation was confirmed by carotenoid extraction and quantification from the seven strains after 24, 72 and $120 \mathrm{~h}$ of cultivation; the pigment composition was analyzed by RP-HPLC (Table 4). The cyp61- mutants produced more carotenoids than their corresponding parental strains without other major alterations in their composition. In all cases, the maximum carotenoid content was reached after $120 \mathrm{~h}$ of cultivation, which coincides with the late stationary phase of growth (Figure 8). In general at this time, the major differences in total carotenoid content were observed among the analyzed strains. The total carotenoid contents relative to the parental strains after 24, 72 and $120 \mathrm{~h}$ of cultivation, respectively, were as follows: $126 \%$, $132 \%$ and $101 \%$ in strain $385-C Y P 61 /$ cyp $^{\text {hph }}$; $179 \%, 217 \%$ and $191 \%$ in strain $385-c y p 61^{\text {hph }} /$ cyp $61^{z e o} ; 116 \%, 153 \%$ and $138 \%$ in strain CBS-cyp61 $1^{\text {hph }}$ and $100 \%, 141 \%$ and $134 \%$ in strain Av2-cyp61 $1^{z e o}$ (Table 4).

\section{Expression analysis of the HMGR gene}

In Schizosaccharomyces pombe, Cryptococcus neoformans and mammalian cells, the expression of the HMG-CoA reductase encoding gene (HMGR) is regulated by sterols $[11,32,33]$. In $X$. dendrorhous, only one HMGR gene [GenBank: AJ884949] has been identified, and its deduced amino acid sequence shares $58 \%$ identity and $73.4 \%$ similarity with HMG1, one of the two HMG-CoA reductases in $S$. cerevisiae [34]. Thus, it is possible that the $X$. dendrorhous cyp $61^{-}$mutants have a higher HMGR transcript level, which could explain the increase in carotenoid content. We quantified the HMGR mRNA level by RT-qPCR at different timepoints on the growth curve of the seven analyzed strains. Figure 8 shows the relative expression of this gene normalized to the housekeeping beta-actin gene [35]. The $H M G R$ gene expression pattern was different between the cyp $61^{-}$mutants and their parental strains. While its expression is relatively constant in the wild-type strains, its expression in the 385-cyp61 $1^{h p h} / c y p 61^{z e o}$, CBS-cyp $61^{h p h}$ and Av2-cyp61 $61^{z e o}$ mutants increases along the growth curve, reaching expression levels almost 23, 12 and 73 times higher, respectively, than the wild-type strains after $120 \mathrm{~h}$ of cultivation. At 24 and $72 \mathrm{~h}$ of cultivation, the expression of this gene was between 2 and 5 times higher in the 385-cyp61 $61^{h p h} /$ cyp $61^{z e o}, \mathrm{CBS}-c y p 61^{\text {hph }}$ and Av2-cyp61 $61^{\text {zeo }}$ strains than in the respective parental strains (Figure 8).

\section{Discussion}

Cytochrome P450 monooxygenases are involved in the oxidative metabolism of an enormous diversity of substrates, taking part in primary, secondary and xenobiotic metabolism. CYP51 and CYP61 are structurally and functionally conserved fungal P450s involved in membrane ergosterol biosynthesis [36], and the role of CYP61 as a
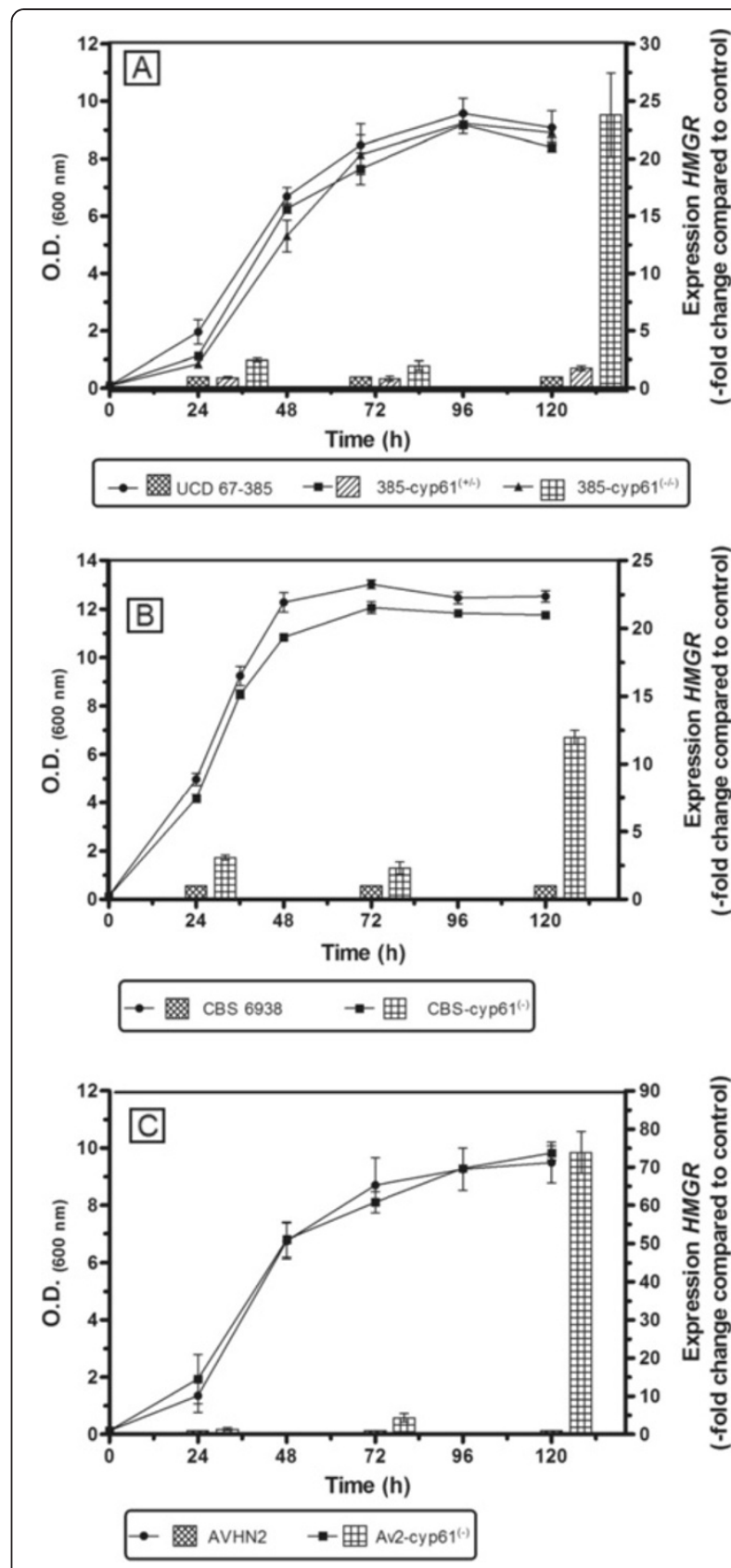

Figure 8 RT-qPCR expression analysis of the HMGR gene along the growth curve in wild-type and cyp61 mutant strains. The HMGR gene expression in the mutant strains was determined with respect to the control (wild-type strain). A) Strains UCD 67-385, 385CYP61/cyp61 hph and 385-cyp61 hph/cyp61 zeo B) CBS 6938 and CBScyp6 $1^{\text {hph }}$. C) AVHN2 and Av2-cyp61zeo. Values are the mean \pm standard error of three independent experiments.

C22-desaturase in fungal membrane sterol synthesis has been elucidated in S. cerevisiae [24] and Candida glabrata [37]. In this study, we isolated and characterized a gene, CYP61, from $X$. dendrorhous that has nine exons, encodes a putative 526-residue polypeptide and shares significant similitude and identity with the C22-sterol desaturase 
from S. cerevisiae [25]. We could predict several P450 characteristic secondary structural elements, and we identified three residues in CYP61 that are completely conserved in P450s. Together, these observations support the hypothesis that the $X$. dendrohous CYP61 gene encodes the cytochrome P450 CYP61.

As in other organisms [25], the CYP61 gene is not essential for the $X$. dendrorhous viability, even though we demonstrated that it is involved in ergosterol biosynthesis. Disruption of the CYP61 gene prevents ergosterol biosynthesis and leads to the accumulation of other intermediary sterols including ergosta-5,8-dien-3-ol and ergosta-5,8,22-trien-3-ol. Contrary to our findings, the specific mutation of ERG5 in S. cerevisiae results in the predominant accumulation of ergosta-5,7-dien-3-ol, although the C22-desaturase substrate is ergosta-5,7,24trien-3-ol [25,38]. Like in $X$. dendrohous, ergosta-5,8, 22-trien-3-ol accumulation has been observed in other fungi, such as $C$. neoformans, after the inhibition of the ERG6-encoding enzyme [39] and in nystatin-resistant Neurospora crassa strains that are unable to produce ergosterol [40]. Although our second found intermediary, ergosta-5,8-dien-3-ol, is an atypical sterol, it has been detected in fungi strains that are unable to synthetize ergosterol that in turn are resistant to fungicidal polyenes, such as nystatin and primaricin; polyenes bind ergosterol in the fungal cell membrane, creating channels that disrupt the transmembrane potential and its functions [41]. This phenomenon was observed in a nystatin-resistant S. cerevisiae strain [42] and primaricin-resistant Aspergillus nidulans strains [43]. Clearly, these observations and our results indicate the existence of alternative sterol biosynthesis pathways, which require further studies.

Because the cyp61- mutant strains were viable without significant changes in their growth (at least in our experimental conditions), ergosta-5,8,22-trien-3-ol and/or ergosta-5,8-dien-3-ol may play roles similar to ergosterol in the $X$. dendrorhous cell membrane. Finally, even though the cyp $61^{-}$mutant strains were not able to produce ergosterol, their sterol content was higher compared to the corresponding parental strains, suggesting an ergosterolmediated feedback regulatory mechanism in the sterol biosynthesis pathway of $X$. dendrorhous.

In addition to the alterations in sterol content and composition, the cyp $61^{-}$mutant $X$. dendrorhous strains exhibited color phenotypes dissimilar to their parental strains (Figure 7). Carotenoid analyses revealed that the mutant strains produced more carotenoids (Table 4), demonstrating that the CYP61 gene mutation affected carotenoid biosynthesis. Major differences were observed after 72 and $120 \mathrm{~h}$ of culture, which coincide with the early and late stationary phases of growth (Figure 8). Wozniak and co-workers reported that maximum expression levels of carotenogenic genes are reached by the end of the exponential and beginning of the stationary phase of $X$. dendrorhous growth [44], coinciding with the induction of carotenogenesis [45]. It is expected that greater differences in the carotenoid content would be observed once carotenogenesis is induced.

Similar to our results, other studies have demonstrated an increase in astaxanthin production in Phaffia rhodozyma (anamorphic state of $X$. dendrorhous) when the ergosterol levels were reduced by fluconazole treatment [46]. A possible explanation for the increased carotenoids in the cyp $61^{-}$mutants could be the greater availability of carotenoid precursors in absence of the ergosterol negative feedback regulation. This reasoning is also supported by the fact that in the cyp $61^{-}$mutants, the total sterol content was also increased. For example, supplementation of $P$. rhodozyma cultures with MVA resulted in an increase in carotenoid production [47]. Likewise, deletion of the squalene synthase-encoding gene (ERG9) in combination with the overexpression of the catalytic domain of HMGR in a recombinant $C$. utilis strain that produces carotenoids caused an increase of in lycopene biosynthesis [48].

IPP is the isoprenoid building block; in most eukaryotes, it is derived from the MVA pathway [10]. Many of the regulatory aspects of isoprenoid biosynthesis involve elements of this pathway; the expression of $H M G R$ (Figure 1) is a critical regulatory step [49]. The alteration of HMGR expression in the X. dendrorhous cyp $61^{-} \mathrm{mu}-$ tants could explain the increased carotenoid and sterol content. We quantified the HMGR transcript levels, and at all of the growth phases analyzed, it was greater than in the corresponding parental strain. Similarly, an increase in the HMGR transcript level corresponded to an increase in the carotenoid content when the fungus Blaskelea tripora was treated with ketoconazole, which is a specific ergosterol biosynthesis inhibitor [50]. In $S$. cerevisiae, HMG-CoA reductase is encoded by two isogenes, $H M G 1$ and $H M G 2$, and the expression of $H M G 1$ is controlled at the transcriptional level by ergosterol [26]. The overexpression of HMG1 combined with ketoconazole treatment in a $S$. cerevisiae recombinant strain resulted in an increase in beta-carotene production [51]. Finally, our results are similar to those reported in the astaxanthin over-producing $X$. dendrorhous mutant strain with lower ergosterol and a higher HMGR transcript level than the parental strain after $72 \mathrm{~h}$ of cultivation [46]. However, the astaxanthin over-producing strain was obtained by random chemical mutagenesis, while we specifically blocked ergosterol biosynthesis by disrupting the CYP61 gene.

\section{Conclusions}

In conclusion, the CYP61 gene disruption in $X$. dendrorhous prevents the synthesis of ergosterol without 
affecting the growth of the yeast under the experimental conditions used in this work. The cyp $61^{-}$mutant strains accumulate ergosta-5,8,22-trien-3-ol and ergosta-5,8dien-3-ol that may fulfill some of the ergosterol roles in the cell. In addition, our results strongly suggest that by a feedback regulatory mechanism, ergosterol regulates the synthesis of sterols and carotenoids in the astaxanthinproducing yeast $X$. dendrorhous, being the HMGR gene expression, one of its targets.

\section{Methods}

\section{Microorganisms, plasmids, media, and enzymes}

The strains and plasmids that were used or created in this work are listed in Table 2. The wild-type UCD 67$385 X$. dendrorhous strain was used for cDNA library construction and genomic CYP61 gene amplification. E. coli $\mathrm{DH}-5 \alpha$ was used as a host for plasmid propagation.

$X$. dendrorhous strains were grown at $22^{\circ} \mathrm{C}$ with constant agitation in YM medium ( $1 \%$ glucose, $0.3 \%$ yeast extract, $0.3 \%$ malt extract and $0.5 \%$ peptone). Yeast transformant selection was performed on 1.5\% agar YMplates supplemented with $10 \mu \mathrm{g} / \mathrm{ml}$ hygromycin B and/ or $15 \mu \mathrm{g} / \mathrm{ml}$ zeocin. E. coli strains were grown with constant agitation at $37^{\circ} \mathrm{C}$ in Luria-Bertani (LB) medium supplemented with $100 \mu \mathrm{g} / \mathrm{ml}$ ampicillin for plasmid selection and $40 \mu \mathrm{l}$ of a $2 \%$ solution of X-gal (5-bromo4chloro-3-indolyl- $\beta$-D-galactopyranoside) for recombinant clone selection [52]. Recombinant clones bearing the plasmids with the hygromycin B or zeocin resistance cassettes were selected by direct colony PCR with primers specific for each cassette [21,31]. The zeocin resistance cassette was constructed in the same way as the hygromycin B resistance cassette [31] using the Sh ble gene from Streptoalloteichus hindustanus [53,54]. The Taq DNA polymerase (pol), restriction enzymes, Klenow polymerase and M-MLV reverse transcriptase were purchased from Promega, and the Pfu DNA pol was purchased from Invitrogen.

\section{DNA amplification and sequence analysis}

The oligonucleotides designed for this study (Table 1) were purchased from Alpha DNA or from Integrated DNA Technologies. PCR reactions were performed in a DNA thermal cycler 2400 (Perkin-Elmer) in a final volume of $25 \mu \mathrm{l}$ containing $2 \mathrm{U}$ of Taq DNA pol, $2.5 \mu \mathrm{l}$ of 10X Taq buffer, $0.5 \mu \mathrm{l}$ of $10 \mathrm{mM}$ dNTPs, $1 \mu \mathrm{l}$ of $50 \mathrm{mM}$ $\mathrm{MgCl}_{2}, 1 \mu \mathrm{l}$ of each primer $(25 \mu \mathrm{M})$ and 10 to $20 \mathrm{ng}$ of template DNA. In general, the amplification protocol was as follows: initial denaturation at $95^{\circ} \mathrm{C}$ for $3 \mathrm{~min} ; 35$ cycles of denaturation at $94^{\circ} \mathrm{C}$ for $30 \mathrm{~s}$, annealing at $55^{\circ} \mathrm{C}$ for $30 \mathrm{~s}$, and synthesis at $72^{\circ} \mathrm{C}$ for $3 \mathrm{~min}$; and a final extension step at $72^{\circ} \mathrm{C}$ for $10 \mathrm{~min}$. Samples were kept at $4^{\circ} \mathrm{C}$ until checked by $0.8 \%$ agarose gel electrophoresis in TAE buffer containing $0.5 \mu \mathrm{g} / \mathrm{ml}$ ethidium bromide [52]. DNA for sequencing or plasmid construction was purified from gels with glass milk [55].

Nucleotide sequences were obtained from an ABI 3100 Avant genetic analyzer using the BigDye terminator v3.1 kit (Applied Biosystems). DNA sequences were analyzed with Vector NTI Suite 10 (Informax), CLUSTAL W 1.8 and programs available at the NCBI web site. Protein sequence analyses were performed with programs available at www.ch.embnet.org/software/TMPRED_form.html [56], www.ebi.ac.uk/InterProScan/ [57] and www.cyped. uni-stuttgart.de/ [58].

\section{Cloning of the X. dendrorhous CYP61 gene and plasmid construction}

Our group has partially sequenced the genome of the wild-type UCD 67-385 X. dendrorhous strain by two Next Generation Sequencing (NGS) systems. Our collection of scaffolds covers approximately $95 \%$ of the haploid genome of the yeast. We used the CLC Genomics Workbench 5 for genome analyses. BLAST analyses allowed us to identify the $X$. dendrorhous CYP61 gene, and primers were designed from its sequence (Table 1).

The pBS-gCyp61 plasmid (Figure 4) was generated by inserting a 4,224 bp PCR-amplified DNA fragment encoding the CYP61 gene into the EcoRV site of pBluescript SK- plasmid. The DNA fragment was amplified using the primer set CYP61up2.F + CYP61dw2.R (Table 1) and genomic DNA of the UCD 67-385 wild-type strain as template. Plasmids pBS-cyp61/Hyg and pBS-cyp61/Zeo were created by cloning the hygromycin $\mathrm{B}$ and the zeocin resistance cassettes, respectively, into the EcoRV site of plasmid pBS-cyp61 (Figure 4). Plasmid pBS-cCyp61, bearing the cDNA of the CYP61 gene, was obtained from a X. dendrorhous cDNA library made with the pBluescript II XR cDNA library construction kit (Stratagene) [31].

\section{$X$. dendrorhous transformation}

$X$. dendrorhous transformation was performed by electroporation according to [59] and [60]. Electrocompetent cells were prepared from an exponential culture $\left(\mathrm{OD}_{600 \mathrm{~nm}}=\right.$ 1.2), grown in YM medium and electroporated using a BioRad gene pulser $\times$ cell with PC and CE modules under the following conditions: $125 \mathrm{mF}, 600 \Omega, 0.45 \mathrm{kV}$. Transformations were performed using 1 to $5 \mu \mathrm{g}$ of linear donor DNA prepared by cutting pBS-cyp61/Hyg or pBS-cyp61/ Zeo with $\mathrm{XbaI}$. The transformant strains were identified as $X$. dendrorhous by analysis of the ITS1, 5.8 rRNA gene and ITS2 DNA sequences [61]. The transformant strains were identified as $X$. dendrorhous by analysis of the ITS1, 5.8 rRNA gene and ITS2 DNA sequences [61].

\section{Phenotypic analyses of cyp61 mutant strains}

To compare the phenotypic differences between wildtype and CYP61 mutant strains, phenotypic analyses were 
performed. The strains were grown in YM medium, and growth curves were constructed including the analyses of total carotenoid yield and composition, ergosterol production and relative mRNA expression of the HMGR gene at three timepoints. For these analyses, the seven $X$. dendrorhous strains (UCD 67-385, 385-cyp61 ${ }^{(+/-)}$, 385-cyp61 $1^{(-/-)}$, CBS 6938, CBS-cyp61 ${ }^{(-)}$, AVHN2 and Av2-cyp61 $1^{(-)}$) were cultivated in triplicate $600 \mathrm{ml} \mathrm{YM}$ cultures in Erlenmeyer flasks at $22^{\circ} \mathrm{C}$ with constant agitation. The yeast growth was determined by the OD at $600 \mathrm{~nm}$, which was measured in V-630 UV-vis Spectrophotometer from JASCO. Culture samples of $75 \mathrm{ml}$ were taken after 24, 72 and 120 $\mathrm{h}$ of growth and segregated for analysis as follows: $5 \mathrm{ml}$ to determine the dry weight of the yeast, $30 \mathrm{ml}$ for RNA, 30 $\mathrm{ml}$ for pigment and $10 \mathrm{ml}$ for sterol extractions. In each case, the cell pellet was washed with distilled water, frozen with liquid nitrogen and stored at $-80^{\circ} \mathrm{C}$ until further processing.

\section{Carotenoid extraction and RP-HPLC}

Carotenoids were extracted from cellular pellets according to the acetone extraction method [62]. Total carotenoids were quantified by absorbance at $465 \mathrm{~nm}$ using an absorption coefficient of $\mathrm{A}_{1 \%}=2,100$ and normalized to the dry weight of the yeast. Carotenoids were separated by RP-HPLC using a reverse phase RP-18 Lichrocart125-4 (Merck) column with acetonitrile: methanol: isopropyl $(85: 10: 5 \mathrm{v} / \mathrm{v})$ as the mobile phase with a $1 \mathrm{ml} /$ min flux under isocratic conditions. The elution spectra were recovered using a diode array detector, and carotenoids were identified by their spectra and retention time according to standards.

\section{Sterol extraction and identification}

Sterol extraction was adapted from [63] and [64]. Briefly, $4 \mathrm{~g}$ of $\mathrm{KOH}$ and $16 \mathrm{ml}$ of $60 \%(\mathrm{v} / \mathrm{v})$ ethanol/water were added to the cell pellets, which were mixed and saponified at $80 \pm 2^{\circ} \mathrm{C}$ for $2 \mathrm{~h}$. Non-saponificable sterols were extracted with $10 \mathrm{ml}$ of petroleum and dried. Sterols were separated by RP-HPLC with a C-18 column, using metha$\mathrm{nol} /$ water $(97: 3, \mathrm{v} / \mathrm{v})$ as the mobile phase at $1 \mathrm{ml} / \mathrm{min}$. The elution spectra were recovered using a diode array detector, and sterols were visualized in the $280 \mathrm{~nm}$ channel. Standard ergosterol was purchased at Sigma-Aldrich (catalogue number 57-87-4). Sterols were quantified spectrophotometrically at $280 \mathrm{~nm}$ [65]. The identification of the sterols was performed by an external service (Corthorn Quality; http://www.corthorn.cl/) by GC/MS (Agilent $5970 \mathrm{~N}$ gas chromatographer/Agilent $5890 \mathrm{~N}$ mass spectrometer). An RTX5 sil MS (Restk) $30 \mathrm{~m} \times 250 \mu \mathrm{m} \times$ $0.25 \mu \mathrm{m}$ column was used with the following oven conditions: $270^{\circ} \mathrm{C}$ for $10 \mathrm{~s}$, raised to $280^{\circ} \mathrm{C}$ at $30^{\circ} \mathrm{C} / \mathrm{min}$ and maintained for $2 \mathrm{~min}$. The injector temperature was $270^{\circ} \mathrm{C}$, and the ion source was kept at $70 \mathrm{eV}$. Helium was used as the mobile phase with a flux rate of $1 \mathrm{ml} / \mathrm{min}$. For sterol identification the NIST Standard Reference Database 1A (NIST/EPA/NIH Mass Spectral Library (NIST 08) and NIST Mass Spectral Search Program version 2.0f, was used (http://www.nist.gov/srd/).

\section{RNA extraction, single strand DNA synthesis and RT-qPCR}

Total RNA extraction from the cell pellets was performed via mechanical rupture with $0.5 \mathrm{~mm}$ glass beads (BioSpec) and shaking in a vortex apparatus for $10 \mathrm{~min}$ followed by the addition of Tri-Reagent (Ambion). The lysate was incubated for $10 \mathrm{~min}$ at room temperature, and $150 \mu \mathrm{l}$ of chloroform per $\mathrm{ml}$ of Tri-Reagent was added. The aqueous phase was recovered after centrifugation for $5 \mathrm{~min}$ at $4,000 \mathrm{x} \mathrm{g}$. Two consecutive extractions with acidic phenol:chloroform (1:1) were performed, and the RNA was precipitated by adding two volumes of isopropanol and incubating at room temperature for $10 \mathrm{~min}$. The RNA was washed with $75 \%$ ethanol, suspended in RNase-free $\mathrm{H}_{2} \mathrm{O}$ and quantified by absorbance determination at $260 \mathrm{~nm}$ in V-630 UV-vis Spectrophotometer from JASCO.

The synthesis of cDNA was performed according to the M-MLV reverse transcriptase (Invitrogen) manufacturer's protocol, with $5 \mu \mathrm{g}$ of total RNA in a final volume of $20 \mu \mathrm{l}$. The determination of the relative gene expression levels was performed in an Mx3000P quantitative PCR system (Stratagene) using $1 \mu \mathrm{l}$ of the reverse transcription reaction, $0.25 \mu \mathrm{M}$ of each primer (Table 1) and $10 \mu \mathrm{l}$ of the SensiMix SYBR Green I (Quantace) kit in a final volume of $20 \mu \mathrm{l}$. The $\mathrm{Ct}$ values obtained were normalized to the respective value of the beta-actin, $A C T$ [Genbank: X89898.1] [66] and later expressed as a function of the control conditions using the $\Delta \Delta \mathrm{Ct}$ algorithm [35].

\section{Additional file}

Additional file 1: Figure S1. GC-MS analysis of sterols from wild-type and cyp61 X. dendrorhous mutant strain. GC profiles of sterols (peaks № 1, 2 and 3) from UCD 67-385 (panel A) and 385-cyp61 $1^{(-/)}$(panel B) strains. Sterols structures were identified according to their retention times and mass spectra (NIST Standard Reference Database). Panels C, $D$ and $E$ show the sample (in red) and Database (in blue) mass spectra: ergosterol (peak № 1, panel C), ergosta-5,8,22-trien-3-ol (peak № 2, panel D) and ergosta-5,8-dien-3-ol (peak № 3, panel E).

\section{Competing interests}

The authors declare no competing financial or any non-financial interests.

\section{Authors' contributions}

JA conceived the study, participated in its design and coordination. JA carried out the cyp61 gene isolation, sequence analysis and X. dendrorhous transformation. IL performed the gene expression, pigment and ergosterol extraction analyses. MSG did the genomic transformants analyses and SB accomplished the growth curves of wild-type and cyp61 mutant strains. DS participated in DNA sequencing. PM-M participated in the gene expression analyses. MB contributed in the study design. VC participated in the 
experiment design and coordination. JA, MB, VC drafted the manuscript. All authors read and approved the final manuscript.

\section{Acknowledgements}

This work was supported by projects: U. de Chile VID Iniciacion I 10/01-2 to JA and Fondecyt 1100324 to VC. MECESUP-604 by a graduate scholarship to IL.

Received: 3 August 2012 Accepted: 12 October 2012

Published: 18 October 2012

\section{References}

1. Golubev Wl: Perfect state of Rhodomyces dendrorhous (Phaffia rhodozyma). Yeast 1995, 11:101-110.

2. Johnson EA: Phaffia rhodozyma: colorful odyssey. Int Microbiol 2003, 6:169-174.

3. Guerin M, Huntley ME, Olaizola M: Haematococcus astaxanthin: applications for human health and nutrition. Trends Biotechnol 2003, 21:210-216.

4. Schroeder WA, Johnson EA: Antioxidant role of carotenoids in Phaffia rhodozyma. J Gen Microbiol 1993, 139:907-912.

5. Schroeder WA, Johnson EA: Singlet oxygen and peroxyl radicals regulate carotenoid biosynthesis in Phaffia rhodozyma. J Biol Chem 1995, 270:18374-18379.

6. Schroeder WA, Johnson EA: Carotenoids protect Phaffia rhodozyma against singlet oxygen damage. J Ind Microbiol Biotechnol 1995, 14:502-507.

7. Fassett RG, Coombes JS: Astaxanthin: a potential therapeutic agent in cardiovascular disease. Mar Drugs 2011, 9:447-465.

8. Higuera-Ciapara I, Felix-Valenzuela L, Goycoolea FM: Astaxanthin: a review of its chemistry and applications. Crit Rev Food Sci Nutr 2006, 46:185-196.

9. Britton G, Liaaen-Jensen S, Pfander H: Carotenoids handbook. Switzerland: Birkhäuser Verlag; 2004

10. Miziorko HM: Enzymes of the mevalonate pathway of isoprenoid biosynthesis. Arch Biochem Biophys 2011, 505:131-143.

11. Goldstein $J \mathrm{~L}$, Brown MS: Regulation of the mevalonate pathway. Nature 1990, 343:425-430.

12. Merkulov S, van Assema F, Springer J, Fernandez del Carmen A, Mooibroek $\mathrm{H}$ : Loning and characterization of the Yarrowia lipolytica squalene synthase (SQS1) gene and functional complementation of theSaccharomyces cerevisiae erg9 mutation. Yeast 2000, 16:197-206.

13. Verdoes JC, Krubasik P, Sandmann G, Van Ooyen AJJ: Isolation and functional characterization of a novel type of carotenoid biosynthetic gene from Xanthophyllomyces dendrorhous. Mol Gen Genet 1999, 262:453-461.

14. Verdoes JC, Misawa N, van Ooyen AJJ: Cloning and characterization of the astaxanthin biosynthetic gene encoding phytoene desaturase of Xanthophyllomyces dendrorhous. Biotechnol Bioeng 1999, 63:750-755.

15. Ojima K, Breitenbach J, Visser H, Setoguchi Y, Tabata K, Hoshino T, van den Berg J, Sandmann G: Cloning of the astaxanthin synthase gene from Xanthophyllomyces dendrorhous (Phaffia rhodozyma) and its assignment as a $\beta$-carotene 3-hydroxylase/4-ketolase. Mol Genet Genomics 2006, 275:148-158.

16. Álvarez V, Rodríguez-Sáiz M, de la Fuente JL, Gudiña EJ, Godio RP, Martín JF, Barredo JL: The crtS gene of Xanthophyllomyces dendrorhous encodes a novel cytochrome-P450 hydroxylase involved in the conversion of [beta]-carotene into astaxanthin and other Xanthophylls. Fungal Genet Biol 2006, 43:261-272.

17. Zhang H, Im SC, Waskell L: Cytochrome b5 increases the rate of product formation by cytochrome P450 2B4 and competes with cytochrome $\mathrm{P} 450$ reductase for a binding site on cytochrome P450 2B4. J Biol Chem 2007, 282:29766-29776.

18. Degtyarenko KN, Archakov Al: Molecular evolution of P450 superfamily and P450-containing monooxygenase systems. FEBS Lett 1993, 332:1-8.

19. Kimmich N, Das A, Sevrioukova I, Meharenna Y, Sligar SG, Poulos TL: Electron transfer between cytochrome P450cin and its FMN-containing redox partner, cindoxin. J Biol Chem 2007, 282:27006-27011.

20. McLean KJ, Sabri M, Marshall KR, Lawson RJ, Lewis DG, Clift D, Balding PR, Dunford AJ, Warman AJ, McVey JP: Biodiversity of cytochrome P450 redox systems. Biochem Soc Trans 2005, 33:796-801.

21. Alcaíno J, Barahona S, Carmona M, Lozano C, Marcoleta A, Niklitschek M, Sepúlveda D, Baeza M, Cifuentes V: Cloning of the cytochrome p450 reductase $(c r t R)$ gene and its involvement in the astaxanthin biosynthesis of Xanthophyllomyces dendrorhous. BMC Microbio/ 2008, 8:169.

22. Masamoto K, Misawa N, Kaneko T, Kikuno R, Toh H: Beta-carotene hydroxylase gene from the cyanobacterium Synechocystis sp. PCC6803. Plant Cell Physiol 1998, 39:560-564.

23. Zhang YQ, Rao R: Beyond ergosterol: linking $\mathrm{pH}$ to antifungal mechanisms. Virulence 2010, 1:551-554.

24. Kelly SL, Lamb DC, Baldwin BC, Corran AJ, Kelly DE: Characterization of Saccharomyces cerevisiae CYP61, sterol $\Delta 22$-desaturase, and inhibition by azole antifungal agents. J Biol Chem 1997, 272:9986-9988.

25. Skaggs BA, Alexander JF, Pierson CA, Schweitzer KS, Chun KT, Koegel C, Barbuch R, Bard M: Cloning and characterization of the Saccharomyces cerevisiae C-22 sterol desaturase gene, encoding a second cytochrome P-450 involved in ergosterol biosynthesis. Gene 1996, 169:105-109.

26. Veen M, Lang C: Production of lipid compounds in the yeast Saccharomyces cerevisiae. Appl Environ Microbiol 2004, 63:635-646.

27. Werck-Reichhart D, Feyereisen R: Cytochromes P450: a success story. Genome Biol 2000, 1:3003.1-3003.9.

28. Sirim D, Wagner F, Lisitsa A, Pleiss J: The cytochrome P450 engineering database: integration of biochemical properties. BMC Biochem 2009, 10:27.

29. van den Brink $H$, van Gorcom RFM, van den Hondel CA, Punt PJ: Cytochrome P450 enzyme systems in fungi. Fungal Genet Biol 1998, 23:1-17

30. Hermosilla G, Martínez C, Retamales P, León R, Cifuentes V: Genetic determination of ploidy level in Xanthophyllomyces dendrorhous. Antonie Van Leeuwenhoek 2003, 84:279-287.

31. Niklitschek M, Alcaíno J, Barahona S, Sepúlveda D, Lozano C, Carmona M, Marcoleta A, Martínez C, Lodato P, Baeza M: Genomic organization of the structural genes controlling the astaxanthin biosynthesis pathway of Xanthophyllomyces dendrorhous. Biol Res 2008, 41:93-108.

32. Chang YC, Bien CM, Lee H, Espenshade PJ, Kwong-Chung KJ: Sre1p, A regulator of oxygen sensing and sterol homeostasis, is required for virulence in Cryptococcus neoformans. Molec Microbiol 2007, 64:614-629.

33. Hughes AL, Todd BL, Espenshade PJ: SREBP pathway responds to sterols and functions as an oxygen sensor in fission yeast. Cell 2005, 120:831-842.

34. Pearson WR, Wood T, Zhang Z, Miller W: Comparison of DNA sequences with protein sequences. Genomics 1997, 46:24-36.

35. Livak KJ, Schmittgen TD: Analysis of relative gene expression data using real-time quantitative PCR and the 2-[Delta][Delta] CT method. Methods 2001, 25:402-408.

36. Cresnar B, Petric S: Cytochrome P450 enzymes in the fungal kingdom. Biochim Biophys Acta 2011, 1814:29-35.

37. Lamb DC, Maspahy S, Kelly DE, Manning NJ, Geber A, Bennett JE, Kelly SL: Purification, reconstitution, and inhibition of cytochrome P-450 sterol $\triangle 22$-desaturase from the pathogenic fungus Candida glabrata. Antimicrob Agents Chemother 1999, 43:1725-1728.

38. Kristan K, Rizner TL: Steroid-transforming enzymes in fungi. J Steroid Biochem Mol Biol 2012, 129:79-91.

39. Nes WD, Zhou W, Ganapathy K, Liu JL, Vatsyayan R, Chamala S, Hernandez K, Miranda M: Sterol 24-C-methyltransferase: an enzymatic target for the disruption of ergosterol biosynthesis and homeostasis in Cryptococcus neoformans. Arch Biochem Biophys 2009, 481:210-218.

40. Morris DC, Safe S, Subden RE: Detection of the ergosterol and episterol isomers lichesterol and fecosterol in nystatin-resistant mutants of Neurospora crassa. Biochem Genet 1974, 12:459-466.

41. Kanafani ZA, Perfect JR: Antimicrobial resitance: resistance to antifungal agents: mechanisms and clinical impact. Clin Infect Dis 2008, 46:120-128.

42. Shingo $H$, Yoshihisa ODA, Nishino T, Katsuki H, Aoyama Y, Yoshtoa Y, Nagai $\mathrm{J}$ : Characterization of a Saccharomyces cerevisiae mutant, N22, defective in ergosterol synthesis and preparation of [28-14C] ergosta-5, 7-dien-3 $\beta$ ol with the mutant. J Biochem 1983, 94:501-510.

43. Ziogas BN, Sisler HD, Lusby WR: Sterol content and other characteristics of pimaricin-resistant mutants of Aspergillus nidulans. Pestic Biochem Physiol 1983, 20:320-329.

44. Wozniak A, Lozano C, Barahona S, Niklitschek M, Marcoleta A, Alcaíno J, Sepulveda D, Baeza M, Cifuentes V: Differential carotenoid production and gene expression in Xanthophyllomyces dendrorhous grown in a nonfermentable carbon source. FEMS Yeast Res 2011, 11:252-262.

45. Lodato P, Alcaíno J, Barahona S, Niklitschek M, Carmona M, Wozniak A, Baeza M, Jiménez A, Cifuentes V: Expression of the carotenoid 
biosynthesis genes in Xanthophyllomyces dendrorhous. Biol Res 2007, 40:73-84.

46. Miao L, Chi S, Tang Y, Su Z, Yin T, Guan G, Li Y: Astaxanthin biosynthesis is enhanced by high carotenogenic gene expression and decrease of fatty acids and ergosterol in a Phaffia rhodozyma mutant strain. FEMS Yeast Res 2011, 11:192-201.

47. Calo P, Miguel T, Velázquez JB, Villa TG: Mevalonic acid increases transastaxanthin and carotenoid biosynthesis in Phaffia rhodozyma. Biotechnol Lett 1995, 17:575-578,

48. Shimada H, Kondo K, Fraser PD, Miura Y, Saito T, Misawa N: Increased carotenoid production by the food yeast Candida utilis through metabolic engineering of the isoprenoid pathway. Appl Environ Microbiol 1998, 64:2676-2680

49. Parks LW, Casey WM: Physiological implications of sterol biosynthesis in yeast. Annu Rev Microbiol 1995, 49:95-116.

50. Tang Q, Li Y, Yuan QP: Effects of an ergosterol synthesis inhibitor on gene transcription of terpenoid biosynthesis in Blakeslea trispora. Curr Microbiol 2008, 57:527-531.

51. Yan G, Wen K, Duan C: Enhancement of $\beta$-carotene production by overexpression of HMG-CoA reductase coupled with addition of ergosterol biosynthesis inhibitors in recombinant Saccharomyces cerevisiae. Curr Microbiol 2012, 64:1-5.

52. Sambrook J, Russell DW: Molecular cloning. A laboratory manual. 3rd edition. Cold Spring Harbor NY: Cold Spring Harbor Laboratory Press; 2001.

53. Drocourt D, Calmels T, Reynes JP, Baron M, Tiraby G: Cassettes of the Streptoalloteichus hindustanus ble gene for transformation of lower and higher eukaryotes to phleomycin resistance. Nucleic Acids Res 1990, 18:4009-4009

54. Calmels T, Parriche M, Durand H, Tiraby G: High efficiency transformation of Tolypocladium geodes conidiospores to phleomycin resistance. Curr Genet 1991, 20:309-314.

55. Boyle JS, Lew AM: An inexpensive alternative to glassmilk for DNA purification. TIG 1995, 11:8

56. Hofmann K, Stoffel W: TMbase-A database of membrane spanning protein segments. Biol Chem Hoppe Seyler 1993, 374:166.

57. Zdobnov EM, Apweiler R: InterProScan-an integration platform for the signature-recognition methods in InterPro. Bioinformatics 2001 $17: 847-848$

58. Sirim D, Widmann $M$, Wagner F, Pleiss J: Prediction and analysis of the modular structure of cytochrome P450 monooxygenases. BMC Struct Biol 2010, 10:34.

59. Adrio JL, Veiga M: Transformation of the astaxanthin-producing yeast Phaffia rhodozyma. Biotechnol Tech 1995, 9:509-512.

60. Kim IG, Nam SK, Sohn JH, Rhee SK, An GH, Lee SH, Choi ES: Cloning of the ribosomal protein L41 gene of Phaffia rhodozyma and its use as a drug resistance marker for transformation. Appl Environ Microbiol 1998, 64:1947-1949

61. Fell JW, Blatt GM: Separation of strains of the yeasts Xanthophyllomyces dendrorhous and Phaffia rhodozyma based on rDNA IGS and ITS sequence analysis. J Ind Microbiol Biotechnol 1999, 23:677-681.

62. An GH, Schuman DB, Johnson EA: Isolation of Phaffia rhodozyma mutants with increased astaxanthin content. Appl Environ Microbiol 1989, 55:116-124

63. Shang $F$, Wen $S$, Wang $X$, Tan $T$ : Effect of nitrogen limitation on the ergosterol production by fed-batch culture of Saccharomyces cerevisiae. J Biotechnol 2006, 122:285-292.

64. Cheng B, Yuan Q, Sun X, Li W: Enhanced production of coenzyme Q10 by overexpressing HMG-CoA reductase and induction with arachidonic acid in Schizosaccharomyces pombe. Appl Biochem Biotechnol 2010, 160:523531.

65. Lamacka M, Sajbidor J: Ergosterol determination in Saccharomyces cerevisiae comparison of different methods. Biotechnol Tech 1997, 11:723-725.

66. Wery J, Dalderup MJM, Ter Linde J, Boekhout T, Van Ooyen AJJ: Structural and phylogenetic analysis of the actin gene from the yeast Phaffia rhodozyma. Yeast 1996, 12:641-651

\section{Submit your next manuscript to BioMed Central and take full advantage of:}

- Convenient online submission

- Thorough peer review

- No space constraints or color figure charges

- Immediate publication on acceptance

- Inclusion in PubMed, CAS, Scopus and Google Scholar

- Research which is freely available for redistribution 